\title{
Front Matter: Volume 8447
}

, "Front Matter: Volume 8447," Proc. SPIE 8447, Adaptive Optics Systems III, 844701 (13 September 2012); doi: 10.1117/12.2000148

SPIE Event: SPIE Astronomical Telescopes + Instrumentation, 2012, Amsterdam, Netherlands 


\title{
PROCEEDINGS OF SPIE
}

\section{Adaptive Optics Systems III}

\author{
Brent L. Ellerbroek \\ Enrico Marchetti \\ Jean-Pierre Véran \\ Editors
}

1-6 July 2012

Amsterdam, Netherlands

Sponsored by

SPIE

Cooperating Organizations

American Astronomical Society (United States)

Netherlands Institute for Radio Astronomy (ASTRON) (Netherlands)

Ball Aerospace \& Technologies Corporation (United States)

Canadian Astronomical Society (CASCA) (Canada)

European Astronomical Society (Switzerland)

European Southern Observatory (Germany)

International Astronomical Union

Korea Astronomy and Space Science Institute (KASI) (Republic of Korea)

National Radio Astronomy Observatory

POPSud (France)

TNO (Netherlands)

Published by

SPIE

Volume 8447

Part One of Four Parts 
The papers included in this volume were part of the technical conference cited on the cover and title page. Papers were selected and subject to review by the editors and conference program committee. Some conference presentations may not be available for publication. The papers published in these proceedings reflect the work and thoughts of the authors and are published herein as submitted. The publisher is not responsible for the validity of the information or for any outcomes resulting from reliance thereon.

Please use the following format to cite material from this book:

Author(s), "Title of Paper," in Adaptive Optics Systems III, edited by Brent L. Ellerbroek, Enrico Marchetti, Jean-Pierre Véran, Proceedings of SPIE Vol. 8447 (SPIE, Bellingham, WA, 2012) Article CID Number.

ISSN: 0277-786X

ISBN: 9780819491480

Published by

SPIE

P.O. Box 10, Bellingham, Washington 98227-0010 USA

Telephone +1 3606763290 (Pacific Time) · Fax +1 3606471445

SPIE.org

Copyright (C) 2012, Society of Photo-Optical Instrumentation Engineers.

Copying of material in this book for internal or personal use, or for the internal or personal use of specific clients, beyond the fair use provisions granted by the U.S. Copyright Law is authorized by SPIE subject to payment of copying fees. The Transactional Reporting Service base fee for this volume is $\$ 18.00$ per article (or portion thereof), which should be paid directly to the Copyright Clearance Center (CCC), 222 Rosewood Drive, Danvers, MA 01923. Payment may also be made electronically through CCC Online at copyright.com. Other copying for republication, resale, advertising or promotion, or any form of systematic or multiple reproduction of any material in this book is prohibited except with permission in writing from the publisher. The CCC fee code is 0277-786X/12/\$18.00.

Printed in the United States of America.

Publication of record for individual papers is online in the SPIE Digital Library.

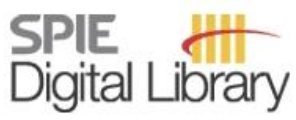

SPIEDigitallibrary.org

Paper Numbering: Proceedings of SPIE follow an e-First publication model, with papers published first online and then in print and on CD-ROM. Papers are published as they are submitted and meet publication criteria. A unique, consistent, permanent citation identifier (CID) number is assigned to each article at the time of the first publication. Utilization of CIDs allows articles to be fully citable as soon as they are published online, and connects the same identifier to all online, print, and electronic versions of the publication. SPIE uses a six-digit CID article numbering system in which:

- The first four digits correspond to the SPIE volume number.

- The last two digits indicate publication order within the volume using a Base 36 numbering

system employing both numerals and letters. These two-number sets start with 00, 01, 02, 03, 04, $05,06,07,08,09,0 \mathrm{~A}, 0 \mathrm{~B} \ldots \mathrm{OZ}$, followed by 10-1Z, 20-2Z, etc.

The CID Number appears on each page of the manuscript. The complete citation is used on the first page, and an abbreviated version on subsequent pages. Numbers in the index correspond to the last two digits of the six-digit CID Number. 


\title{
Contents
}

\section{Part One}

\author{
xxxv Conference Committee \\ xxxix The cosmic microwave background: observing directly the early universe (Plenary Paper) \\ [8442-506] \\ P. de Bernardis, S. Masi, Univ. degli Studi di Roma La Sapienza (Italy)
}

\section{SESSION $1 \quad$ PROJECT STATUS I}

844702 Status of the ARGOS ground layer adaptive optics system [8447-1]

W. Gässler, Max-Planck-Institut für Astronomie (Germany); S. Rabien, Max-Planck-Institut für extraterrestrische Physik (Germany); S. Esposito, INAF - Osservatorio Astrofisico di Arcetri (Italy); M. Lloyd-Hart, The Univ. of Arizona (United States); L. Barl, Max-Planck-Institut für extraterrestrische Physik (Germany); U. Beckmann, Max-Planck-Institut für Radioastronomie (Germany); T. Bluemchen, Max-Planck-Institut für Astronomie (Germany); M. Bonaglia, INAF - Osservatorio Astrofisico di Arcetri (Italy); J. L. Borelli, Max-Planck-Institut für Astronomie (Germany); G. Brusa, J. Brynnel, Large Binocular Telescope Observatory (United States); P. Buschkamp, Max-Planck-Institut für extraterrestrische Physik (Germany); L. Busoni, L. Carbonaro, INAF - Osservatorio Astrofisico di Arcetri (Italy); C. Connot, R. Davies, M. Deysenroth, Max-Planck-Institut für Radioastronomie (Germany); O. Durney, The Univ. of Arizona (United States); R. Green, Large Binocular Telescope Observatory (United States); H. Gemperlein, Max-Planck-Institut für extraterrestrische Physik (Germany); V. Gasho, The Univ. of Arizona (United States); M. Haug, Max-Planck-Institut für extraterrestrische Physik (Germany); P. Hubbard, The Univ. of Arizona (United States); S. Ihle, PNSensor GmbH (Germany); M. Kulas, Max-Planck-Institut für Astronomie (Germany); R. Lederer, MaxPlanck-Institut für extraterrestrische Physik (Germany); J. Lewis, The Univ. of Arizona (United States); C. Loose, Max-Planck-Institut für extraterrestrische Physik (Germany); M. Lehmitz, Max-Planck-Institut für Astronomie (Germany); J. Noenickx, The Univ. of Arizona (United States); E. Nussbaum, Max-Planck-Institut für Radioastronomie (Germany); G. Orban de Xivry, Max-Planck-Institut für extraterrestrische Physik (Germany); D. Peter, Max-Planck-Institut für Astronomie (Germany); A. Quirrenbach, Landessternwarte Heidelberg (Germany); M. Rademacher, The Univ. of Arizona (United States); W. Raab, Max-Planck-Institut für extraterrestrische Physik (Germany); J. Storm, Leibniz-Institut für Astrophysik Potsdam (Germany); C. Schwab, Landessternwarte Heidelberg (Germany); V. Vaitheeswaran, The Univ. of Arizona (United States); J. Ziegleder, Max-Planck-Institut für extraterrestrische Physik (Germany) 
844704 Robo-AO: autonomous and replicable laser-adaptive-optics and science system [8447-4] C. Baranec, R. Riddle, Caltech Optical Observatories (United States); A. N. Ramaprakash, Inter-Univ. Ctr. for Astronomy and Astrophysics (India); N. Law, Dunlap Institute for Astronomy and Astrophysics, Univ. of Toronto (Canada); S. Tendulkar, S. Kulkarni, R. Dekany, K. Bui, J. Davis, Caltech Optical Observatories (United States); M. Burse, H. Das, Inter-Univ. Ctr. for Astronomy and Astrophysics (India); S. Hildebrandt, Caltech Optical Observatories (United States); S. Punnadi, Inter-Univ. Ctr. for Astronomy and Astrophysics (India); R. Smith, Caltech Optical Observatories (United States)

\section{SESSION 2 WAVEFRONT CORRECTORS I}

844705 Overview of deformable mirror technologies for adaptive optics and astronomy (Invited Paper) [8447-5]

P.-Y. Madec, European Southern Observatory (Germany)

844706 TMT DMs final design and advanced prototyping results at Cilas [8447-6]

J.-C. Sinquin, A. Bastard, CILAS (France); C. Boyer, Thirty Meter Telescope Observatory Corp. (United States); S. Cornette, R. Cousty, CILAS (France); B. Ellerbroek, Thirty Meter Telescope Observatory Corp. (United States); X. Gilbert, B. Gourdet, R. Grasser,

D. Groeninck, C. Guillemard, CILAS (France); G. Herriot, NRC Herzberg Institute of Astrophysics (Canada); A. Iannacone, A. Jeulin, A. Moreau, H. Pagès, CILAS (France); L. Wang, Thirty Meter Telescope Observatory Corp. (United States)

844707 Low-cost unimorph deformable mirror with high actuator count for astronomical adaptive optics [8447-7]

J. Ma, Y. Liu, Univ. of Science and Technology of China (China); C. Xu, Nanjing Institute of Astronomical Optics \& Technology (China); H. Rong, B. Li, J. Chu, Univ. of Science and Technology of China (China)

844708 The actuator design and the experimental tests of a new technology large deformable mirror for visible wavelengths adaptive optics [8447-8]

C. Del Vecchio, G. Agapito, C. Arcidiacono, L. Carbonaro, INAF - Osservatorio Astrofisico di Arcetri (Italy); F. Marignetti, E. De Santis, Univ. degli Studi di Cassino (Italy); V. Biliotti,

A. Riccardi, INAF - Osservatorio Astrofisico di Arcetri (Italy)

\section{SESSION 3 QUANTITATIVE ASTRONOMY AND SCIENCE WITH AO I}

8447 OA Adaptive optics observations of the galactic center young stars (Invited Paper) [8447-10] S. Yelda, A. M. Ghez, Univ. of California, Los Angeles (United States); J. R. Lu, Institute for Astronomy, Univ. of Hawai'i (United States); T. Do, Univ. of California, Irvine (United States); L. Meyer, M. R. Morris, Univ. of California, Los Angeles (United States)

8447 OB Adaptive optics for high contrast imaging (Invited Paper) [8447-1 1] M. Kasper, European Southern Observatory (Germany)

8447 OC Quantitative solar system science with AO systems (Invited Paper) [8447-12] F. Marchis, SETI Institute (United States); J. Berthier, IMCCE, Observatoire de Paris, Avenue Denfert-Rochereau (France); M. H. Wong, Univ. of California, Berkeley (United States) 


\section{SESSION 4 LASER SYSTEMS}

8447 OD Progress in laser guide star adaptive optics and lessons learned (Invited Paper) [8447-13] P. Wizinowich, W. M. Keck Observatory (United States)

8447 OE An overview of guidestar laser technologies (Invited Paper) [8447-14]

D. T. Gavel, Univ. of California Observatories (United States)

8447 OF RFA-based 589-nm guide star lasers for ESO VLT: a paradigm shift in performance, operational simplicity, reliability, and maintenance [8447-15]

A. Friedenaver, TOPTICA Photonics AG (Germany); V. Karpov, D. Wei, MPB

Communications Inc. (Canada); M. Hager, B. Ernstberger, TOPTICA Photonics AG

(Germany); W. R. L. Clements, MPB Communications Inc. (Canada); W. G. Kaenders,

TOPTICA Photonics AG (Germany)

8447 OG Towards a practical sodium guide star laser source: design for $>\mathbf{5 0}$ watt LGS based on OPSL [8447-16]

J. D. Berger, J. L. A. Chilla, S. Govorkov, J. F. P. van Nunen, A. Y. Lepert, Coherent, Inc. (United States)

$8447 \mathrm{OH} \quad$ Simulations of pulsed sodium laser guide stars: an overview [8447-17]

R. Holzlöhner, European Southern Observatory (Germany); S. M. Rochester, Rochester

Scientific, LLC (United States) and Univ. of California, Berkeley (United States);

D. Bonaccini Calia, European Southern Observatory (Germany); D. Budker, Univ. of California, Berkeley (United States) and Rochester Scientific, LLC (United States);

T. Pfrommer, European Southern Observatory (Germany); J. M. Higbie, Bucknell Univ.

(United States)

\section{SESSION 5 PROJECT STATUS II}

8447 Ol GeMS: first on-sky results (Invited Paper) [8447-18]

F. Rigaut, Gemini Observatory (Chile) and Research School of Astronomy and Astrophysics, The Australian National Univ. (Australia); B. Neichel, M. Boccas, Gemini Observatory (Chile) C. d'Orgeville, Gemini Observatory (Chile) and Research School of Astronomy and Astrophysics, The Australian National Univ. (Australia); G. Arriagada, V. Fesquet, S. J. Diggs, C. Marchant, G. Gausachs, W. N. Rambold, J. Luhrs, S. Walker, E. R. Carrasco-Damele, M. L. Edwards, P. Pessev, R. L. Galvez, T. B. Vucina, C. Araya, A. Gutierrez, A. W. Ebbers, A. Serio, C. Moreno, C. Urrutia, R. Rogers, R. Rojas, C. Trujillo, B. Miller, D. A. Simons, A. Lopez, V. Montes, H. Diaz, F. Daruich, F. Colazo, Gemini Observatory (Chile); M. Bec, G. Trancho, M. Sheehan, Giant Magellan Telescope Organization Corp. (United States); P. McGregor, P. J. Young, M. C. Doolan, J. van Harmelen, Research School of Astronomy and Astrophysics, The Australian National Univ. (Australia); B. L. Ellerbroek, Thirty Meter Telescope Observatory Corp. (United States); D. Gratadour, LESIA - Observatoire de Paris (France);

A. Garcia-Rissmann, European Southern Observatory (Germany)

8447 OJ ESO adaptive optics facility progress report (Invited Paper) [8447-19]

R. Arsenault, P.-Y. Madec, J. Paufique, P. La Penna, S. Stroebele, E. Vernet, J.-F. Pirard, W. Hackenberg, H. Kuntschner, L. Jochum, J. Kolb, N. Muller, M. Le Louarn, P. Amico, N. Hubin, J.-L. Lizon, R. Ridings, J. A. Abad, G. Fischer, V. Heinz, M. Kiekebusch,

J. Argomedo, R. Conzelmann, S. Tordo, R. Donaldson, C. Soenke, P. Duhoux, E. Fedrigo, 
B. Delabre, A. Jost, M. Duchateau, M. Downing, J. R. Moreno, R. Dorn, A. Manescau, D. Bonaccini Calia, M. Quattri, C. Dupuy, I. M. Guidolin, M. Comin, R. Guzman, B. Buzzoni, J. Quentin, S. Lewis, P. Jolley, M. Kraus, T. Pfrommer, European Southern Observatory (Germany); R. Biasi, Microgate S.r.l. (Italy); D. Gallieni, A.D.S. International S.r.l. (Italy); C. Bechet, Ctr. de Recherche Astronomique de Lyon (France); R. Stuik, Leiden Observatory (Netherlands)

8447 OK Tests of open-loop LGS tomography with CANARY (Invited Paper) [8447-20] T. J. Morris, A. G. Basden, Durham Univ. (United Kingdom); F. Vidal, Observatoire de Paris (France); A. P. Reeves, Durham Univ. (United Kingdom); E. Gendron, Observatoire de Paris (France); R. M. Myers, Durham Univ. (United Kingdom); Z. Hubert, Observatoire de Paris (France); E. J. Younger, Durham Univ. (United Kingdom); A. Longmore, UK Astronomy Technology Ctr. (United Kingdom); M. Cohen, Observatoire de Paris (France); N. Dipper, P. Clark, Durham Univ. (United Kingdom); D. Henry, UK Astronomy Technology Ctr. (United Kingdom); G. C. Rousset, Observatoire de Paris (France); S. P. Todd, UK Astronomy Technology Ctr. (United Kingdom); F. Chemla, Observatoire de Paris (France); D. C. Atkinson, UK Astronomy Technology Ctr. (United Kingdom); J.-M. Huet, Observatoire de Paris (France); B. Stobie, C. J. Dickson, UK Astronomy Technology Ctr. (United Kingdom)

8447 OL Image quality and high contrast improvements on VLT/NACO [8447-21]

J. H. V. Girard, J. O'Neal, European Southern Observatory (Chile); D. Mawet, European Southern Observatory (Chile) and Jet Propulsion Lab. (United States); M. Kasper, European Southern Observatory (Germany); G. Zins, IPAG, Univ. Joseph Fourier, CNRS (France); B. Neichel, Gemini Observatory (Chile); J. Kolb, European Southern Observatory (Germany); V. Christiaens, Univ. de Liège (Belgium) and European Southern Observatory (Chile); M. Tourneboeuf, Univ. Católica de Chile (Chile)

SESSION 6 QUANTITATIVE ASTRONOMY AND SCIENCE WITH AO II

8447 OM Science with ESO's Multi-conjugate Adaptive-optics Demonstrator - MAD (Invited Paper) [8447-22]

J. Melnick, E. Marchetti, P. Amico, European Southern Observatory (Germany)

8447 ON Results from the commissioning of the Gemini South Adaptive Optics Imager (GSAOI) at Gemini South Observatory [8447-23]

E. R. Carrasco, M. L. Edwards, Gemini Observatory (Chile); P. J. McGregor, Research School of Astronomy and Astrophysics, The Australian National Univ. (Australia); C. Winge, Gemini Observatory (Chile); P. J. Young, M. C. Doolan, J. van Harmelen, Research School of Astronomy and Astrophysics, The Australian National Univ. (Australia); F. J. Rigaut, B. Neichel, Gemini Observatory (Chile); G. Trancho, Giant Magellan Telescope Organization Corp. (United States); E. Artigau, Univ. de Montréal (Canada); P. Pessev, F. Colazo, Gemini Observatory (Chile); J. Tigner, Univ. of Victoria (Canada); F. Mauro, Univ. de Concepción (Chile); J. Lührs, W. N. Rambold, Gemini Observatory (Chile)

$844700 \quad$ High-contrast imaging in the Hyades with snapshot LOCI [8447-160]

K. M. Morzinski, Steward Observatory, The Univ. of Arizona (United States); B. A. Macintosh, Lawrence Livermore National Lab. (United States); L. M. Close, Steward Observatory, The Univ. of Arizona (United States); C. Marois, NRC Herzberg Institute of Astrophysics (Canada); Q. Konopacky, Dunlap Institute for Astronomy and Astrophysics (Canada); J. Patience, School of Earth \& Space Exploration, Arizona State Univ. (United States) 
8447 OP Theoretical limits on bright star astrometry with multi-conjugate adaptive optics using a diffractive pupil [8447-25]

S. M. Ammons, Lawrence Livermore National Lab. (United States); E. A. Bendek, Steward Observatory, The Univ. of Arizona (United States); O. Guyon, Steward Observatory, The Univ. of Arizona (United States) and Subaru Telescope (United States); B. Macintosh, D. Savransky, Lawrence Livermore National Lab. (United States)

\section{SESSION 7 WAVEFRONT SENSING I}

$8447 \mathrm{OQ}$ Advances in defector technologies for visible and infrared wavefront sensing (Invited Paper) [8447-26]

P. Feautrier, Institut de Planétologie et d'Astrophysique de Grenoble, UJF-Grenoble I, CNRSINSU (France) and First Light Imaging (France); J.-L. Gach, Observatoire Astronomique de Marseille-Provence (France) and First Light Imaging (France); M. Downing, European Southern Observatory (Germany); P. Jorden, e2v technologies (United Kingdom); J. Kolb, European Southern Observatory (Germany); J. Rothman, CEA-LETI-Minatec (France); T. Fusco, ONERA (France); P. Balard, Observatoire Astronomique de Marseille-Provence (France) and First Light Imaging (France); E. Stadler, Institut de Planétologie et d'Astrophysique de Grenoble, UJF-Grenoble I, CNRS-INSU (France) and First Light Imaging (France); C. Guillaume, Observatoire de Haute-Provence (France) and First Light Imaging (France); D. Boutolleau, First Light Imaging (France); G. Destefanis, N. Lhermet, CEA-LETIMinatec (France); O. Pacaud, M. Vuillermet, A. Kerlain, SOFRADIR (France); N. Hubin, J. Reyes, M. Kasper, O. Ivert, European Southern Observatory (Germany); W. Suske, A. Walker, M. Skegg, e2v technologies (United Kingdom); S. Derelle, J. Deschamps, C. Robert, N. Vedrenne, ONERA (France); F. Chazalet, SHAKTI (France); J. Tanchon, T. Trollier, A. Ravex, Absolut Systems (France); G. Zins, P. Kern, T. Moulin, O. Preis, Institut de Planétologie et d'Astrophysique de Grenoble, UJF-Grenoble I, CNRS-INSU (France)

8447 OR Measured performance of the prototype polar coordinate CCD array (Invited Paper) [8447-27]

S. M. Adkins, W. M. Keck Observatory (United States)

8447 OT The AOLI low-order non-linear curvature wavefront sensor: a method for high sensitivity wavefront reconstruction [8447-29]

J. Crass, P. Aisher, Institute of Astronomy, Univ. of Cambridge (United Kingdom); B. Femenia, Instituto de Astrofísica de Canarias (Spain) and Univ. Politécnica de Cartagena (Spain); D. L. King, C. D. Mackay, Institute of Astronomy, Univ. of Cambridge (United Kingdom); R. Rebolo-López, Instituto de Astrofísica de Canarias (Spain) and Consejo Superior de Investigaciones Científicas (Spain); L. Labadie, I. Physikalsiches Institut, Univ. zu Köln (Germany); A. Pérez Garrido, Univ. Politécnica de Cartagena (Spain); M. Balcells, Isaac Newton Group of Telescopes (Spain), Instituto de Astrofísica de Canarias (Spain), and Univ. de La Laguna (Spain); A. Díaz Sánchez, Univ. Politécnica de Cartagena (Spain); J. J. Fuensalida, Instituto de Astrofísica de Canarias (Spain) and Univ. de La Laguna (Spain); R. L. Lopez, Instituto de Astrofísica de Canarias (Spain); A. Oscoz, J. A. Pérez Prieto, Instituto de Astrofísica de Canarias (Spain) and Univ. de La Laguna (Spain); L. F. Rodríguez-Ramos, Instituto de Astrofísica de Canarias (Spain); I. Villó, Univ. Politécnica de Cartagena (Spain) 
8447 OU Natural guide star adaptive optics systems at LBT: FLAO commissioning and science operations status [8447-30]

S. Esposito, A. Riccardi, E. Pinna, A. T. Puglisi, F. Quirós-Pacheco, INAF - Osservatorio Astrofisico di Arcetri (Italy); C. Arcidiacono, INAF - Osservatorio Astronomico di Bologna (Italy); M. Xompero, R. Briguglio, L. Busoni, L. Fini, J. Argomedo, A. Gherardi, G. Agapito, INAF - Osservatorio Astrofisico di Arcetri (Italy); G. Brusa, D. L. Miller, J. C. Guerra, K. Boutsia, LBT Observatory, The Univ. of Arizona (United States); P. Stefanini, INAF - Osservatorio Astrofisico di Arcetri (Italy)

8447 OV LINC-NIRVANA Pathfinder: testing the next generation of wave front sensors at LBT [8447-121]

A. R. Conrad, Max-Planck-Institut für Astronomie (Germany); C. Arcidiacono, INAF Osservatorio Astronomico di Bologna (Italy); H. Baumeister, Max-Planck-Institut für Astronomie (Germany); M. Bergomi, INAF - Osservatorio Astronomico di Padova (Italy); T. Bertram, J. Berwein, Max-Planck-Institut für Astronomie (Germany); C. Biddick, Large Binocular Telescope Observatory (United States); P. Bizenberger, M. Brangier, F. Briegel, Max-Planck-Institut für Astronomie (Germany); A. Brunelli, INAF - Osservatorio Astronomico di Padova (Italy); J. Brynnel, Large Binocular Telescope Observatory (United States); L. Busoni, INAF - Osservatorio Astrofisico di Arcetri (Italy); N. Cushing, Large Binocular Telescope Observatory (United States); F. De Bonis, Max-Planck-Institut für Astronomie (Germany); M. De La Pena, Large Binocular Telescope Observatory (United States); S. Esposito, INAF - Osservatorio Astrofisico di Arcetri (Italy); J. Farinato, INAF - Osservatorio Astronomico di Padova (Italy); L. Fini, INAF - Osservatorio Astrofisico di Arcetri (Italy); R. F. Green, Large Binocular Telescope Observatory (United States); T. Herbst, R. Hofferbert, F. Kittmann, M. Kuerster, W. Laun, D. Meschke, L. Mohr, A. Pavlov, J.-U. Pott, Max-PlanckInstitut für Astronomie (Germany); A. Puglisi, INAF - Osservatorio Astrofisico di Arcetri (Italy); R. Ragazzoni, INAF - Osservatorio Astronomico di Padova (Italy); A. Rakich, Large Binocular Telescope Observatory (United States); R.-R. Rohloff, J. Trowitzsch, Max-Planck-Institut für Astronomie (Germany); V. Viotto, INAF - Osservatorio Astronomico di Padova (Italy); X. Zhang, Max-Planck-Institut für Astronomie (Germany)

8447 OW Science readiness of the Gemini MCAO system: GeMS [8447-32]

B. Neichel, Gemini Observatory (Chile); F. Rigaut, Research School of Astronomy and Astrophysics, The Australian National Univ. (Australia); A. Serio, G. Arriagada, M. Boccas, Gemini Observatory (Chile); C. d'Orgeville, Research School of Astronomy and Astrophysics, The Australian National Univ. (Australia); V. Fesquet, C. Trujillo, W. N. Rambold, R. L. Galvez, G. Gausachs, T. B. Vucina, V. Montes, C. Urrutia, C. Moreno, S. J. Diggs, C. Araya, J. Lührs, Gemini Observatory (Chile); G. Trancho, M. Bec, Giant Magellan Telescope Organization Corp. (United States); C. Marchant, F. Collao, E. R. Carrasco, M. L. Edwards, P. Pessev, A. Lopez, H. Diaz, Gemini Observatory (Chile)

8447 OX First closed-loop visible AO test results for the advanced adaptive secondary AO system for the Magellan Telescope: MagAO's performance and status [8447-33]

L. M. Close, J. R. Males, D. Kopon, V. Gasho, K. B. Follette, P. Hinz, K. Morzinski, Steward Observatory, The Univ. of Arizona (United States); A. Uomoto, T. Hare, OCIW (United States); A. Riccardi, S. Esposito, A. Puglisi, E. Pinna, L. Busoni, C. Arcidiacono, M. Xompero, R. Briguglio, F. Quiros-Pacheco, J. Argomedo, INAF - Osservatorio Astrofisico di Arcetri (Italy) (Italy) 
8447 OY Results from the PALM-3000 high-order adaptive optics system [8447-34]

J. E. Roberts, Jet Propulsion Lab. (United States); R. G. Dekany, Caltech Optical

Observatories (United States); R. S. Burruss, Jet Propulsion Lab. (United States); C. Baranec,

Caltech Optical Observatories (United States); A. Bouchez, Giant Magellan Telescope

Organization Corp. (United States); E. E. Croner, S. R. Guiwits, D. D. S. Hale, Caltech Optical Observatories (United States); J. R. Henning, Palomar Observatory, California Institute of Technology (United States); D. L. Palmer, M. Troy, T. N. Truong, Jet Propulsion Lab. (United States); J. Zolkower, Cornell Univ. (United States)

\section{SESSION 9 ADVANCES IN AO CONTROL I}

$84470 Z$ Vibration mitigation in adaptive optics control (Invited Paper) [8447-35]

C. Kulcsár, P. Massioni, L2TI, Institut Galilée, Univ. Paris 13 (France); G. Sivo, L2TI, Institut Galilée, Univ. Paris 13 (France) and ONERA (France); H.-F. Raynaud, L2TI, Institut Galilée, Univ. Paris 13 (France)

844710 Distributed control of large deformable mirrors (Invited Paper) [8447-36]

D. G. MacMartin, California Institute of Technology (United States); R. Heimsten, T. Andersen, M. Owner-Petersen, Lund Univ. (Sweden)

844711 Design of frequency-based controllers for vibration mitigation at the Gemini-South telescope [8447-37]

A. Guesalaga, Univ. Católica de Chile (Chile); B. Neichel, F. Rigaut, Gemini Observatory Southern Operations Ctr. (Chile); J. Osborn, D. Guzman, Univ. Católica de Chile (Chile)

844712 On the rejection of vibrations in adaptive optics systems [8447-38]

R. Muradore, Univ. degli Studi di Verona (Italy); L. Pettazzi, E. Fedrigo, R. Clare, European Southern Observatory (Germany)

\section{SESSION 10 WAVEFRONT SENSING II}

844713 Comparison of LGS wavefront-sensing with pyramid, yaw, and quad-cell types wavefront sensors [8447-39]

E. Gendron, D. Gratadour, LESIA, Observatoire de Paris, CNRS, UPMC, Univ. Paris Dideroł (France)

844714 Wavefront sensing and correction with the Gemini Planet Imager [8447-40]

S. Thomas, Gemini Observatory (United States); L. Poyneer, D. Savransky, Lawrence Livermore National Lab. (United States); B. Macintosh, NRC Herzberg Institute of Astrophysics (Canada); M. Hartung, Gemini Observatory (United States); D. Dillon, D. Gavel, UCO Lick Observatory (United States); J. Dunn, NRC Herzberg Institute of Astrophysics (Canada); K. Wallace, Jet Propulsion Lab. (United States); D. Palmer, Lawrence Livermore National Lab. (United States); R. De Rosa, Univ. of Exeter (United Kingdom)

844715 Focal-plane wave front sensing strategies for high contrast imaging: experimental validations on SPHERE [8447-41]

J.-F. Sauvage, T. Fusco, C. Petit, L. Mugnier, ONERA (France); B. Paul, ONERA (France) and Lab. d'Astrophysique de Marseille (France); A. Costille, IPAG (France) 
844716 Retrieving the telescope and instrument static wavefront aberration with a phase diversity procedure using on-sky adaptive optics corrected images [8447-42]

L. Jolissaint, aquilAOptics (Switzerland); L. M. Mugnier, ONERA (France); C. Neyman,

W. M. Keck Observatory (United States); J. Christou, Gemini Observatory (United States);

P. Wizinowich, W. M. Keck Observatory (United States)

844717 Design of a truth sensor for the GMT laser tomography adaptive optics system [8447-43]

M. A. van Dam, Flat Wavefronts (New Zealand); R. Conan, The Research School of

Astronomy and Astrophysics, The Australian National Univ. (Australia); A. H. Bouchez, Giant Magellan Telescope Organization Corp. (United States); B. Espeland, The Research School of Astronomy and Astrophysics, The Australian National Univ. (Australia)

SESSION 11 AO DISTURBANCES MODELING AND CHARACTERIZATION I

844718 Turbulence modeling and estimation for AO systems (Invited Paper) [8447-44]

A. Beghi, A. Cenerede, A. Masiero, Univ. degli Studi di Padova (Italy)

844719 Mesospheric sodium structure variability on horizontal scales relevant to laser guide star asterisms (Invited Paper) [8447-45]

T. Pfrommer, European Southern Observatory (Germany); P. Hickson, The Univ. of British Columbia (Canada)

8447 1A Lunar scintillometer to validate GLAO turbulence distribution measurements [8447-46] K. Newman, M. Hart, E. Bendek, Ctr. for Astronomical Adaptive Optics, The Univ. of Arizona (United States); E. Bustos, Cerro Tololo Inter-American Observatory (Chile)

8447 1B Estimation of vertical profiles of wind from MASS measurements [8447-47] M. V. Kornilov, Lomonosov Moscow State Univ., Sternberg Astronomical Institute (Russian Federation)

\section{Part Two}

\section{SESSION 12 AO DISTURBANCES MODELING AND CHARACTERIZATION II}

8447 IC Vibrations in AO control: a short analysis of on-sky data around the world [8447-48] C. Kulcsár, L2TI, Institut Galilée, Univ. Paris 13 (France); G. Sivo, L2TI, Institut Galilée, Univ. Paris 13 (France) and ONERA (France); H.-F. Raynaud, L2TI, Institut Galilée, Univ. Paris 13 (France); B. Neichel, F. Rigaut, Gemini Observatory (Chile); J. Christou, Gemini Observatory (United States); A. Guesalaga, Univ. Católica de Chile (Chile); C. Correia, J.-P. Véran, NRC Herzberg Institute of Astrophysics (Canada); E. Gendron, F. Vidal, G. Rousset, LESIA, Observatoire de Paris, CNRS, Univ. Paris Diderot (France); T. Morris, Durham Univ. (United Kingdom); S. Esposito, F. Quiros-Pacheco, G. Agapito, INAF - Osservatorio Astrofisico di Arcetri (Italy); E. Fedrigo, L. Pettazzi, R. Clare, European Southern Observatory (Germany); R. Muradore, Univ. of Verona (Italy); O. Guyon, F. Martinache, Subaru Telescope, National Astronomical Observatory of Japan (United States); S. Meimon, J.-M. Conan, ONERA (France) 
8447 ID Tolerancing the fabrication errors of static optical elements for ELT-size wide-field AO systems [8447-49]

J.-P. Véran, J. Pazder, G. Herriot, D. Andersen, NRC Herzberg Institute of Astrophysics (Canada)

\section{SESSION 13 PROJECT STATUS IV}

$8447 \mathrm{IF}$ Subaru laser guide adaptive optics system: performance and science operation [8447-52] Y. Minowa, Y. Hayano, H. Terada, T.-S. Pyo, S. Oya, M. Hattori, Subaru Telescope, National Astronomical Observatory of Japan (United States); M. Shirahata, Japan Aerospace Exploration Agency (Japan); H. Takami, National Astronomical Observatory of Japan (Japan); O. Guyon, V. Garrel, S. Colley, M. Weber, T. Golota, Subaru Telescope, National Astronomical Observatory of Japan (United States); M. Watanabe, Hokkaido Univ. (Japan); Y. Saito, Tokyo Institute of Technology (Japan); M. Ito, Univ. of Victoria (Canada); M. Iye, National Astronomical Observatory of Japan (Japan)

8447 IG 'Imaka: working towards very wide-field of view AO [8447-2] M. Chun, Univ. of Hawaili, Hilo (United States); O. Lai, J.-C. Cuillandre, Canada-FranceHawaii Telescope Corp. (United States); H. Richer, The Univ. of British Columbia (Canada); D. Toomey, Mauna Kea Infrared LLC (United States); D. Salmon, Canada-France-Hawaii Telescope Corp. (United States); R. Carlberg, Univ. of Toronto (Canada); D. Andersen, NRC Herzberg Institute of Astrophysics (Canada); D. Burgarella, Observatoire Astronomique de Marseille-Provence (France); K. Ho, Canada-France-Hawaii Telescope Corp. (United States); J. Pazder, NRC Herzberg Institute of Astrophysics (Canada); E. Bertin, Institut d'Astrophysique de Paris (France)

\section{SESSION $14 \quad$ AO FOR ELTS}

844711 The Giant Magellan Telescope adaptive optics program [8447-54]

A. H. Bouchez, Giant Magellan Telescope Organization Corp. (United States); D. S. Acton, Ball Aerospace \& Technologies Corp. (United States); G. Agapito, C. Arcidiacono, INAF Osservatorio Astrofisico di Arcetri (Italy); F. Bennet, Research School of Astronomy and Astrophysics, The Australian National Univ. (Australia); V. Biliotti, M. Bonaglia, R. Briguglio, INAF - Osservatorio Astrofisico di Arcetri (Italy); G. Brusa-Zappellini, Steward Observatory, The Univ. of Arizona (United States); L. Busoni, L. Carbonaro, INAF - Osservatorio Astrofisico di Arcetri (Italy); J. L. Codona, Steward Observatory, The Univ. of Arizona (United States); R. Conan, Research School of Astronomy and Astrophysics, The Australian National Univ. (Australia); T. Connors, O. Durney, Steward Observatory, The Univ. of Arizona (United States); B. Espeland, Research School of Astronomy and Astrophysics, The Australian National Univ. (Australia); S. Esposito, L. Fini, INAF - Osservatorio Astrofisico di Arcetri (Italy); R. Gardhouse, Research School of Astronomy and Astrophysics, The Australian National Univ. (Australia); T. M. Gauron, Smithsonian Astrophysical Observatory (United States); M. Hart, P. M. Hinz, Steward Observatory, The Univ. of Arizona (United States);

S. Kanneganti, Smithsonian Astrophysical Observatory (United States); E. J. Kibblewhite, The Univ. of Chicago (United States); R. P. Knox, Steward Observatory, The Univ. of Arizona (United States); B. A. McLeod, Smithsonian Astrophysical Observatory (United States); T. McMahon, M. Montoya, Steward Observatory, The Univ. of Arizona (United States); T. J. Norton, M. P. Ordway, Smithsonian Astrophysical Observatory (United States); C. d'Orgeville, S. Parcell, P. K. Piatrou, Research School of Astronomy and Astrophysics, The 
Australian National Univ. (Australia); E. Pinna, INAF - Osservatorio Astrofisico di Arcetri (Italy); I. Price, Research School of Astronomy and Astrophysics, The Australian National Univ. (Australia); A. Puglisi, F. Quiros-Pacheco, A. Riccardi, INAF - Osservatorio Astrofisico di Arcetri (Italy); J. B. Roll, Smithsonian Astrophysical Observatory (United States); G. Trancho, Giant Magellan Telescope Organization Corp. (United States); K. Uhlendorf, Research School of Astronomy and Astrophysics, The Australian National Univ. (Australia); $\checkmark$. Vaitheeswaran, Steward Observatory, The Univ. of Arizona (United States); M. A. van Dam, Flat Wavefronts (New Zealand); D. Weaver, Smithsonian Astrophysical Observatory (United States); M. Xompero, INAF - Osservatorio Astrofisico di Arcetri (Italy)

8447 iJ TMT adaptive optics program status report [8447-55]

B. L. Ellerbroek, Thirty Meter Telescope Observatory Corp. (United States); S. M. Adkins, W. M. Keck Observatory (United States); D. R. Andersen, J. Atwood, National Research Council Canada (Canada); A. Bastard, CILAS (France); Y. Bo, Technical Institute of Physics and Chemistry (China); M.-A. Boucher, National Research Council Canada (Canada); C. Boyer, Thirty Meter Telescope Observatory Corp. (United States); P. W. G. Byrnes, K. Caputa, National Research Council Canada (Canada); S. Chen, Institute of Optics and Electronics (China); C. Correia, National Research Council Canada (Canada); R. Cousty, CILAS (France); J. T. Fitzsimmons, National Research Council Canada (Canada); L. Gilles, Thirty Meter Telescope Observatory Corp. (United States); J. Gregory, MIT Lincoln Lab. (United States); G. Herriot, National Research Council Canada (Canada); P. Hickson, The Univ. of British Columbia (Canada); A. Hill, J. Pazder, National Research Council Canada (Canada); H. Pagès, CILAS (France); T. Pfrommer, The Univ. of British Columbia (Canada); V. A. Reshetov, S. Roberts, National Research Council Canada (Canada); J.-C. Sinquin, CILAS (France); M. Schoeck, Thirty Meter Telescope Observatory Corp. (United States) and National Research Council Canada (Canada); M. Smith, J.-P. Véran, National Research Council Canada (Canada); L. Wang, Thirty Meter Telescope Observatory Corp. (United States); K. Wei, Institute of Optics and Electronics (China); I. Wevers, National Research Council Canada (Canada)

8447 IK Dual-channel multiple natural guide star wavefront sensor for the E-ELT multiconjugate adaptive optics module [8447-56]

E. Diolaiti, INAF - Osservatorio Astronomico di Bologna (Italy); L. Schreiber, INAF Osservatorio Astronomico di Padova (Italy); I. Foppiani, M. Lombini, INAF - Osservatorio Astronomico di Bologna (Italy)

8447 IL Wavefront sensor design for the GMT natural guide star AO system [8447-57]

S. Esposito, E. Pinna, F. Quirós-Pacheco, A. T. Puglisi, L. Carbonaro, M. Bonaglia, V. Biliotti, R. Briguglio, G. Agapito, INAF - Osservatorio Astrofisico di Arcetri (Italy); C. Arcidiacono, INAF - Osservatorio Astrofisico di Bologna (Italy); L. Busoni, M. Xompero, A. Riccardi, L. Fini, INAF - Osservatorio Astrofisico di Arcetri (Italy); A. Bouchez, Giant Magellan Telescope Organization Corp. (United States)

8447 IM TMT NFIRAOS: adaptive optics system for the Thirty Meter Telescope [8447-58] G. Herriot, D. Andersen, J. Atwood, P. Byrnes, NRC Herzberg Institute of Astrophysics (Canada); M.-A. Boucher, INO (Canada); C. Boyer, Thirty Meter Telescope Observatory Corp. (United States); K. Caputa, C. Correia, J. Dunn, NRC Herzberg Institute of Astrophysics (Canada); B. Ellerbroek, Thirty Meter Telescope Observatory Corp. (United States); J. Fitzsimmons, NRC Herzberg Institute of Astrophysics (Canada); L. Gilles, Thirty Meter 
Telescope Observatory Corp. (United States); P. Hickson, The Univ. of British Columbia (Canada); A. Hill, D. Kerley, J. Pazder, V. Reshetov, S. Roberts, M. Smith, J.-P. Véran, NRC Herzberg Institute of Astrophysics (Canada); L. Wang, Thirty Meter Telescope Observatory Corp. (United States); I. Wevers, NRC Herzberg Institute of Astrophysics (Canada)

\section{SESSION 15 WAVEFRONT SENSING III}

844710 LIFT, a noise-effective low order focal-plane sensor: from theory to full experimental validation [8447-60]

C. Plantet, ONERA (France); B. Neichel, Gemini Southern Observatory (Chile); S. Meimon, T. Fusco, J.-M. Conan, ONERA (France)

\section{SESSION 16 LASER SYSTEM TESTS}

$84471 Q$ Gemini South multi-conjugate adaptive optics (GeMS) laser guide star facility on-sky performance results [8447-62]

C. d'Orgeville, Research School of Astronomy and Astrophysics, The Australian National Univ. (Australia) and Gemini Observatory (Chile); S. Diggs, V. Fesquet, B. Neichel, W. Rambold, Gemini Observatory (Chile); F. Rigaut, Research School of Astronomy and Astrophysics, The Australian National Univ. (Australia) and Gemini Observatory (Chile); A. Serio, C. Araya, G. Arriagada, R. Balladares, Gemini Observatory (Chile); M. Bec, Giant Magellan Telescope Organization Corp. (United States); M. Boccas, C. Duran, A. Ebbers, A. Lopez, C. Marchant, E. Marin, V. Montes, C. Moreno, E. Petit Vega, C. Segura, Gemini Observatory (Chile); G. Trancho, Giant Magellan Telescope Organization Corp. (United States); C. Trujillo, C. Urrutia, P. Veliz, T. Vucina, Gemini Observatory (Chile)

8447 IR Photon returns test of the pulsed sodium guide star laser on the 1.8 meter telescope [8447-63]

K. Wei, Institute of Optics and Electronics (China); Y. Bo, Technical Institute of Physics and Chemistry (China); X. Xue, Univ. of Science and Technology of China (China); X. Cheng, Wuhan Institute of Physics and Mathematics (China); C. Li, Institute of Optics and Electronics (China); J. Zuo, S. Xie, Technical Institute of Physics and Chemistry (China); C. Rao, Y. Zhang, Institute of Optics and Electronics (China)

\section{SESSION 17 ADVANCES IN AO CONTROL II}

8447 is Advanced control of low order modes in laser guide star multi-conjugate adaptive optics systems [8447-64]

C. Correia, J.-P. Véran, G. Herriot, NRC Herzberg Institute of Astrophysics (Canada);

B. Ellerbroek, L. Wang, L. Gilles, Thirty Meter Telescope Observatory Corp. (United States)

8447 IT Ensemble Transform Kalman Filter, a nonstationary control law for complex AO systems on ELTs: theoretical aspects and first simulations results [8447-65]

M. Gray, B. Le Roux, Lab. d'Astrophysique de Marseille, Aix-Marseille Univ., CNRS (France) 
8447 IU Evidence that wind prediction with multiple guide stars reduces tomographic errors and expands MOAO field of regard [8447-66]

S. M. Ammons, L. Poyneer, Lawrence Livermore National Lab. (United States); D. T. Gavel,

R. Kupke, C. E. Max, UCO Lick Observatory, Univ. of California, Santa Cruz (United States);

L. Johnson, National Solar Observatory (United States)

8447 IV Experimental comparison of tomographic control schemes using the ONERA WFAO facility [8447-67]

A. Parisot, ONERA (France) and Lab. d'Astrophysique de Marseille (France); C. Petit, ONERA (France); T. Fusco, ONERA (France) and Lab. d'Astrophysique de Marseille (France); J.-M. Conan, ONERA (France)

\section{SESSION 18 EXTREME AO}

8447 1X How ELTs will acquire the first spectra of rocky habitable planets [8447-69]

O. Guyon, Subaru Telescope, National Astronomical Observatory of Japan (United States), Steward Observatory, Univ. of Arizona (United States), and College of Optical Sciences, Univ. of Arizona (United States); F. Martinache, Subaru Telescope, National Astronomical Observatory of Japan (United States); E. Cady, Jet Propulsion Lab. (United States); R. Belikov, NASA Ames Research Ctr. (United States); K. Balasubramanian, D. Wilson, Jet Propulsion Lab. (United States); C. Clergeon, Subaru Telescope, National Astronomical Observatory of Japan (United States); M. Mateen, College of Optical Sciences, The Univ. of Arizona (United States)

8447 IY The Subaru coronagraphic extreme AO project: first observations [8447-70] F. Martinache, Subaru Telescope, National Astronomical Observatory of Japan (United States); O. Guyon, Subaru Telescope, National Astronomical Observatory of Japan (United States) and The Univ. of Arizona (United States); C. Clergeon, V. Garrel, Subaru Telescope, National Astronomical Observatory of Japan (United States) and Observatoire de ParisMeudon (France); C. Blain, Univ. of Victoria (Canada)

844712 The SPHERE XAO system SAXO: integration, test, and laboratory performance [8447-71] C. Petit, J.-F. Sauvage, ONERA (France); A. Sevin, LESIA (France); A. Costille, IPAG (France); T. Fusco, ONERA (France); P. Baudoz, LESIA (France); J.-L. Beuzit, IPAG (France); T. Buey, LESIA (France); J. Charton, IPAG (France); K. Dohlen, LAM (France); P. Feautrier, IPAG (France); E. Fedrigo, European Southern Observatory (Germany); J.-L. Gach, LAM (France); N. Hubin, European Southern Observatory (Germany); E. Hugot, LAM (France); M. Kasper, European Southern Observatory (Germany); D. Mouillet, IPAG (France); D. Perret, LESIA (France); P. Puget, IPAG (France); J.-C. Sinquin, CILAS (France); C. Soenke, M. Suarez, European Southern Observatory (Germany); F. Wildi, Observatoire de Genève (Switzerland)

844720 Project 1640: the world's first ExAO coronagraphic hyperspectral imager for comparative planetary science [8447-72]

B. R. Oppenheimer, American Museum of Natural History (United States); C. Beichman, California Institute of Technology (United States); D. Brenner, American Museum of Natural History (United States); R. Burruss, E. Cady, Jet Propulsion Lab. (United States); J. Crepp, L. Hillenbrand, S. Hinkley, California Institute of Technology (United States); E. R. Ligon, T. Lockhart, Jet Propulsion Lab. (United States); I. Parry, Institute of Astronomy, Univ. of Cambridge (United Kingdom); L. Pueyo, Johns Hopkins Univ. (United States); E. Rice, American Museum of Natural History (United States); L. C. Roberts, Jr., J. Roberts, M. Shao, 
Jet Propulsion Lab. (United States); A. Sivaramakrishnan, R. Soummer, Space Telescope Science Institute (United States); G. Vasisht, F. Vescelus, J. K. Wallace, C. Zhai, Jet Propulsion Lab. (United States); N. Zimmerman, Max-Planck-Institut für Astronomie (Germany)

$844721 \quad$ Extremely fast focal-plane wavefront sensing for extreme adaptive optics [8447-73] C. U. Keller, V. Korkiakoski, Leiden Observatory (Netherlands); N. Doelman, TNO Science and Industry (Netherlands); R. Fraanje, R. Andrei, M. Verhaegen, Delft Ctr. for Systems and Control (Netherlands)

844722 On advanced estimation techniques for exoplanet detection and characterization using ground-based coronagraphs [8447-74]

P. R. Lawson, Jet Propulsion Lab. (United States); L. Poyneer, Lawrence Livermore National Lab. (United States); H. Barrett, College of Optical Sciences, The Univ. of Arizona (United States); R. Frazin, Univ. of Michigan (United States); L. Caucci, College of Optical Sciences, The Univ. of Arizona (United States); N. Devaney, National Univ. of Ireland, Galway (Ireland); L. Furenlid, College of Optical Sciences, The Univ. of Arizona (United States); S. Gładysz, Fraunhofer Institute (Germany); O. Guyon, Steward Observatory, The Univ. of Arizona (United States) and Subaru Telescope, National Astronomical Observatory of Japan (United States); J. Krist, Jet Propulsion Lab. (United States); J. Maire, David Dunlap Institute, Univ. of Toronto (Canada); C. Marois, NRC Herzberg Institute of Astrophysics (Canada); D. Mawet, European Southern Observatory (Chile); D. Mouillet, Lab. d'Astrophysique de l'Observatoire de Grenoble (France); L. Mugnier, ONERA (France); I. Pearson, College of Optical Sciences, The Univ. of Arizona (United States); M. Perrin, Space Telescope Science Institute (United States); L. Pueyo, Johns Hopkins Univ. (United States); D. Savransky, Lawrence Livermore National Lab. (United States)

SESSION 19 AO MODELING, ANALYSIS, AND SIMULATIONS

844723 Computer simulations and real-time control of ELT AO systems using graphical processing units (Invited Paper) [8447-75]

L. Wang, B. Ellerbroek, Thirty Meter Telescope Project (United States)

844724 Modeling anisoplanatism in the Keck II laser guide star AO system [8447-76]

M. P. Fitzgerald, G. Witzel, Univ. of California, Los Angeles (United States); M. C. Britton, the Optical Sciences Co. (United States); A. M. Ghez, L. Meyer, B. N. Sitarski, C. Cheng, E. E. Becklin, Univ. of California, Los Angeles (United States); R. D. Campbell, W. M. Keck Observatory (United States); T. Do, Univ. of California, Irvine (United States); J. R. Lu, Institute for Astronomy (United States); K. Matthews, Caltech Optical Observatories (United States); M. R. Morris, Univ. of California, Los Angeles (United States); C. R. Neyman, W. M. Keck Observatory (United States); G. A. Tyler, the Optical Sciences Co. (United States); P. L. Wizinowich, W. M. Keck Observatory (United States); S. Yelda, Univ. of California, Los Angeles (United States)

844725 Size of the halo of the adaptive optics PSF [8447-77]

S. Gladysz, Fraunhofer Institute of Optronics, System Technologies and Image Exploitation (Germany); M. Le Louarn, N. Yaitskova, European Southern Observatory (Germany); A. Garcia-Rissmann, European Southern Observatory (Germany) and Lab. Nacional de Astrofísica (Brazil); L. Kann, J. D. Drummond, R. L. Johnson, D. Roskey, Air Force Research Lab. (United States) 
844726 A Fresnel propagation analysis of NFIRAOS/IRIS high-contrast exoplanet imaging capabilities [8447-78]

C. Marois, J.-P. Véran, C. Correia, National Research Council Canada (Canada)

SESSION 20 POST-PROCESSING OF AO DATA

844727 Aperture masking behind AO systems (Invited Paper) [8447-79]

M. J. Ireland, Macquarie Univ. (Australia), MQ Research Ctr. in Astronomy, Astrophysics and Astrophotonics, Macquarie Univ. (Australia), and Australian Astronomical Observatory (Australia)

844728 Adaptive optics point spread function reconstruction project at W. M. Keck Observatory: first results with faint natural guide stars [8447-80]

L. Jolissaint, aquilAOptics (Switzerland); C. Neyman, W. M. Keck Observatory (United

States); J. Christou, Gemini Observatory (United States); P. Wizinowich, W. M. Keck

Observatory (United States)

844729 Tip/tilt point spread function reconstruction for laser guide star multi-conjugate adaptive optics [8447-81]

L. Gilles, Thirty Meter Telescope Observatory Corp. (United States); C. Correia, J.-P. Véran, NRC Herzberg Institute of Astrophysics (Canada); L. Wang, B. L. Ellerbroek, Thirty Meter Telescope Observatory Corp. (United States)

8447 2A Temporal convergence of phase spatial covariance matrix measurements in tomographic adaptive optics [8447-218]

O. Martin, É. Gendron, G. Rousset, F. Vidal, LESIA, Observatoire de Paris, CNRS, Univ. Paris-

Diderot (France)

SESSION 21 ADVANCES IN AO CONTROL III

8447 2B First on-sky calibration of an high order adaptive optics system [8447-83]

E. Pinna, F. Quirós-Pacheco, A. Riccardi, R. Briguglio, A. Puglisi, L. Busoni, INAF - Osservatorio Astrofisico di Arcetri (Italy); C. Arcidiacono, INAF - Osservatorio Astrofisico di Arcetri (Italy) and INAF - Osservatorio Astrofisico di Bologna (Italy); J. Argomedo, INAF - Osservatorio Astrofisico di Arcetri (Italy) and European Southern Observatory (Germany); M. Xompero, INAF - Osservatorio Astrofisico di Arcetri (Italy); E. Marchetti, European Southern Observatory (Germany); S. Esposito, INAF - Osservatorio Astrofisico di Arcetri (Italy)

8447 2C Optimization of adaptive optics correction during observations: algorithms and system parameters identification in closed-loop [8447-84]

C. Béchet, M. Tallon, É. Thiébaut, Univ. Lyon 1, Ctr. de Recherche Astronomique de Lyon, CNRS, Ecole Normale Supérieure de Lyon (France)

8447 2D Calibration strategy of the AOF [8447-85]

J. Kolb, P.-Y. Madec, M. Le Louarn, N. Muller, European Southern Observatory (Germany);

C. Béchet, Ctr. de Recherche Astronomique de Lyon (France) 
8447 2E A high-performance FPGA platform for adaptive optics real-time control [8447-86] H. Zhang, Z. Ljusic, G. Hovey, J.-P. Veran, G. Herriot, National Research Council Canada (Canada); M. Dumas, Lyrtech RD Inc. (Canada)

8447 2F Design and implementation of the PALM-3000 real-time control system [8447-87] T. N. Truong, Jet Propulsion Lab. (United States); A. H. Bouchez, Giant Magellan Telescope Organization Corp. (United States); R. S. Burruss, Jet Propulsion Lab. (United States);

R. G. Dekany, S. R. Guiwits, California Institute of Technology (United States); J. E. Roberts, J. C. Shelton, M. Troy, Jet Propulsion Lab. (United States)

\section{SESSION 22 WAVEFRONT CORRECTORS II}

$84472 G \quad$ VLT deformable secondary mirror: integration and electromechanical tests results [8447-88] R. Biasi, M. Andrighettoni, G. Angerer, C. Mair, D. Pescoller, Microgate S.r.l. (Italy); P. Lazzarini, E. Anaclerio, M. Mantegazza, D. Gallieni, A.D.S. International S.r.l. (Italy); E. Vernet, R. Arsenault, P.-Y. Madec, P. Duhoux, European Southern Observatory (Germany); A. Riccardi, M. Xompero, R. Briguglio, INAF - Osservatorio Astrofisico di Arcetri (Italy); M. Manetti, M. Morandini, Politecnico di Milano (Italy)

$84472 \mathrm{H} \quad$ Manufacturing of glassy thin shell for adaptive optics: results achieved [8447-89] F. Poutriquet, A. Rinchet, J. L. Carel, H. Leplan, E. Ruch, R. Geyl, G. Marque, Sagem Défense Sécurité (France)

\section{SESSION 23 WAVEFRONT SENSING IV}

$844721 \quad$ Global wavefront sensing for extremely large telescopes [8447-90] R. Ragazzoni, INAF - Osservatorio Astronomico di Padova (Italy); M. Bergomi, INAF Osservatorio Astronomico di Padova (Italy) and Univ. degli Studi di Padova (Italy); A. Brunelli, M. Dima, J. Farinato, D. Magrin, INAF - Osservatorio Astronomico di Padova (Italy); L. Marafatto, Univ. degli Studi di Padova (Italy); V. Viotto, INAF - Osservatorio Astronomico di Padova (Italy)

8447 2J An interferometric wavefront sensor for high-sensitivity low-amplitude measurements [8447-91]

N. A. Bharmal, R. M. Myers, A. G. Basden, A. P. Reeves, Durham Univ. (United Kingdom)

8447 2K A phase-shifting Zernike wavefront sensor for the Palomar P3K adaptive optics system [8447-92]

J. K. Wallace, S. Crawford, F. Loya, J. Moore, Jet Propulsion Lab. (United States)

8447 2L Fast computer-free holographic adaptive optics [8447-93]

G. Andersen, F. Ghebremichael, R. Gaddipati, P. Gaddipati, HUA Inc. (United States) 
$84472 \mathrm{M}$ The wavefront correction control system for the Advanced Technology Solar Telescope [8447-94]

E. K. Kinney, K. Richards, L. Johnson, T. R. Rimmele, S. C. Barden, National Solar Observatory (United States)

$84472 \mathrm{~N} \quad$ Adaptive optics real time processing design for the advanced technology solar telescope [8447-95]

K. Richards, National Solar Observatory (United States)

844720 The Robo-AO software: fully autonomous operation of a laser guide star adaptive optics and science system [8447-96]

R. L. Riddle, Caltech Optical Observatories (United States); M. P. Burse, Inter-Univ. Ctr. for Astronomy and Astrophysics (India); N. M. Law, Dunlap Institute for Astronomy and Astrophysics, Univ. of Toronto (Canada); S. P. Tendulkar, C. Baranec, Caltech Optical Observatories (United States); A. R. Rudy, National Central Univ. (Taiwan); M. Sitt, Stanford Univ. (United States);

A. Arya, Mississippi State Univ. (United States); A. Papadopoulos, Aristotle Univ. of Thessaloniki (Greece); A. N. Ramaprakash, Inter-Univ. Ctr. for Astronomy and Astrophysics (India); R. G. Dekany, Caltech Optical Observatories (United States)

8447 2P Recent development in real time control system of Subaru LGSAO-188 [8447-97] M. Hattori, Subaru Telescope, National Astronomical Observatory of Japan (United States); M. Shirahata, Institute of Space and Astronomical Science (Japan); Y. Minowa, S. Oya, Y. Hayano, Subaru Telescope, National Astronomical Observatory of Japan (United States); H. Takami, M. Iye, National Astronomical Observatory of Japan (Japan)

$84472 \mathrm{Q}$ SPARTA for the VLT: status and plans [8447-98] M. Suárez Valles, E. Fedrigo, R. H. Donaldson, C. Soenke, S. Zampieri, R. Bourtembourg, H. Tischer, European Southern Observatory (Germany)

8447 2R FPGA-based real time controller for high order correction in EDIFISE [8447-99] L. F. Rodríguez-Ramos, H. Chulani, Y. Martín, T. Dorta, A. Alonso, J. J. Fuensalida, Instituto de Astrofísica de Canarias (Spain)

$84472 S$ An AO real-time control solution for ELT scale instrumentation and application to EAGLE [8447-100]

A. Basden, N. Dipper, R. Myers, E. Younger, Durham Univ. (United Kingdom)

$84472 \mathrm{~T}$ Operation of the adaptive optics system at the Large Binocular Telescope Observatory [8447-101]

D. L. Miller, J.-C. Guerra, Large Binocular Telescope Observatory (United States);

K. Boutsia, Large Binocular Telescope Observatory (United States) and INAF-Roma (Italy);

L. Fini, J. Argomedo, INAF - Osservatorio Astrofisico di Arcetri (Italy); C. Biddick, Large Binocular Telescope Observatory (United States); G. Agapito, C. Arcidiacono, R. Briguglio, INAF - Osservatorio Astrofisico di Arcetri (Italy); G. Brusa, Large Binocular Telescope Observatory (United States); L. Busoni, S. Esposito, INAF - Osservatorio Astrofisico di Arcetri (Italy); J. Hill, Large Binocular Telescope Observatory (United States); C. Kulesa, D. McCarthy, Steward Observatory, The Univ. of Arizona (United States); E. Pinna, A. T. Puglisi, F. Quiros-Pacheco, A. Riccardi, M. Xompero, INAF - Osservatorio Astrofisico di Arcetri (Italy) 
$84472 \mathrm{U}$ Pupil rotation compensation for LINC-NIRVANA [8447-102]

M. Brangier, A. R. Conrad, T. Bertram, X. Zhang, J. Berwein, F. Briegel, T. M. Herbst, MaxPlanck-Institut für Astronomie (Germany); R. Ragazzoni, INAF - Osservatorio Astronomico di Padova (Italy)

$84472 V \quad F O A M:$ the modular adaptive optics framework [8447-103]

T. I. M. van Werkhoven, L. Homs, Leiden Observatory (Netherlands); G. Sliepen, AlbaNova Univ. Ctr. (Sweden); M. Rodenhuis, C. U. Keller, Leiden Observatory (Netherlands)

8447 2Y First laboratory validation of LQG control with the CANARY MOAO pathfinder [8447-106] G. Sivo, L2TI, Institut Galilée, Univ. Paris 13 (France) and ONERA (France); H.-F. Raynaud, L2TI, Institut Galilée, Univ. Paris 13 (France); J.-M. Conan, ONERA (France); C. Kulcsár, L2TI, Institut Galilée, Univ. Paris 13 (France); É. Gendron, F. Vidal, LESIA - Observatoire de Meudon (France); A. Basden, Durham Univ. (United Kingdom)

$84472 Z$ Performance of LQG-based control for AO: a numerical analysis [8447-107] J.-P. Folcher, M. Carbillet, Lab. Lagrange, Univ. de Nice Sophia-Antipolis, CNRS, Observatoire de la Côte d'Azur (France); A. Abelli, Lab. Lagrange, Univ. de Nice SophiaAntipolis, CNRS, Observatoire de la Côte d'Azur (France) and Univ. di Roma (Italy); A. Ferrari, Lab. Lagrange, Univ. de Nice Sophia-Antipolis, CNRS, Observatoire de la Côte d'Azur (France)

844731 Infinite impulse response modal filtering in visible adaptive optics [8447-109] G. Agapito, INAF - Osservatorio Astrofisico di Arcetri (Italy); C. Arcidiacono, INAF Osservatorio Astronomico di Bologna (Italy); F. Quirós-Pacheco, A. Puglisi, S. Esposito, INAF Osservatorio Astrofisico di Arcetri (Italy)

844732 On application of constrained receding horizon control in astronomical adaptive optics [8447-110]

M. V. Konnik, J. De Doná, J. S. Welsh, The Univ. of Newcastle (Australia)

844733 Analysis and experimental demonstration of adaptive optics based on the modal control optimization [8447-111]

B. Li, C. Li, Nanjing Institute of Astronomical Optics \& Technology (China); P. Jia, Nanjing Univ. (China); S. Zhang, Nanjing Institute of Astronomical Optics \& Technology (China)

844734 Mitigation of transient meteor events in sodium layer by TMT NFIRAOS [8447-1 12]

G. Herriot, C. Irvin, NRC Herzberg Institute of Astrophysics (Canada)

844735 Experimental test of turbulence prediction algorithms [8447-1 13]

R. Piazzesi, M. Stangalini, D. Del Moro, F. Berrilli, Univ. degli Studi di Roma Tor Vergata (Italy) 


\section{Part Three}

POSTER SESSION: STATUS OF CURRENT AO INSTRUMENT PROJECTS

844737 GALACSI system design and analysis [8447-115]

S. Ströbele, P. La Penna, R. Arsenault, R. D. Conzelmann, B. Delabre, M. Duchateau, R. Dorn, E. Fedrigo, N. Hubin, J. Quentin, P. Jolley, M. Kiekebusch, J. P. Kirchbauer, B. Klein, J. Kolb, H. Kuntschner, M. Le Louarn, J. L. Lizon, P.-Y. Madec, L. Pettazzi, C. Soenke, S. Tordo, J. Vernet, European Southern Observatory (Germany); R. Muradore, Univ. of Verona (Italy)

844738 Status of the GRAAL system development: very wide-field correction with 4 laser guidestars [8447-116]

J. Paufique, J. Argomedo, R. Arsenault, R. Conzelmann, R. Donaldson, N. Hubin, L. Jochum, A. Jost, M. Kiekebusch, J. Kolb, H. Kuntschner, M. Le Louarn, P.-Y. Madec, R. Siebenmorgen, S. Tordo, European Southern Observatory (Germany)

8447 3A Deploying the testbed for the VLT adaptive optics facility: ASSIST [8447-1 18]

R. Stuik, Leiden Observatory (Netherlands) and NOVA-ASTRON (Netherlands); P. La Penna, C. Dupuy, European Southern Observatory (Germany); M. de Haan, NOVA-ASTRON (Netherlands); R. Arsenault, European Southern Observatory (Germany); W. Boland, NOVA (Netherlands); E. Elswijk, R. ter Horst, NOVA-ASTRON (Netherlands); N. Hubin, P.-Y. Madec, European Southern Observatory (Germany); F. Molster, NOVA (Netherlands); E. Wiegers, Leiden Observatory (Netherlands)

8447 3B Infrared differential imager and spectrograph for SPHERE: performance status with extreme adaptive optics before shipment to ESO/VLT [8447-119]

M. Langlois, CNRS, Ctr. de Recherche Astrophysique de Lyon, Univ. Lyon 1, Observatoire de Lyon (France); A. Vigan, C. Moutou, K. Dohlen, Lab. d'Astrophysique de Marseille, CNRS, Univ. de Provence (France); A. Costille, Institut de Planétologie et d'Astrophysique de Grenoble, CNRS, Univ. Joseph Fourier (France); D. Le Mignant, Lab. d'Astrophysique de Marseille, CNRS, Univ. de Provence (France); P. Martinez, D. Mouillet, Institut de Planétologie et d'Astrophysique de Grenoble, CNRS, Univ. Joseph Fourier (France); A. Boccaletti, LESIA - Observatoire de Paris (France); O. Moeller-Nilsson, Max-Planck-Institut für Astronomie (Germany); J.-F. Sauvage, L. Mugnier, ONERA (France); M. Feldt, MaxPlanck-Institut für Astronomie (Germany); C. Gry, Lab. d'Astrophysique de Marseille, CNRS, Univ. de Provence (France); F. Wildi, Observatoire de Genève, Univ. de Genève (Switzerland); J.-L. Beuzit, Institut de Planétologie et d'Astrophysique de Grenoble, CNRS, Univ. Joseph Fourier (France)

8447 3D Status update and closed-loop performance of the Magellan adaptive optics VisAO camera [8447-122]

D. Kopon, L. M. Close, J. Males, V. Gasho, K. Morzinski, K. Follette, Steward Observatory, The Univ. of Arizona (United States)

$84473 E \quad$ Increasing sky coverage with the Gemini North ALTAIR/LGS AO system [8447-123] J. C. Christou, Gemini Observatory (United States); M. Boccas, Gemini Observatory (Chile); A. Ebbers, R. M. McDermid, Gemini Observatory (United States); R. Oram, Gemini Observatory (Chile); C. Trujillo, B. Walls, Gemini Observatory (United States) 
8447 3F Status of the Raven MOAO science demonstrator [8447-124]

D. R. Andersen, NRC Herzberg Institute of Astrophysics (Canada); C. Bradley, O. Lardière, C. Blain, Univ. of Victoria (Canada); C. Correia, NRC Herzberg Institute of Astrophysics (Canada); R. Desmarais, D. Gamroth, M. Ito, K. Jackson, P. Lach, R. Nash, L. Pham, Univ. of Victoria (Canada); J.-P. Véran, NRC Herzberg Institute of Astrophysics (Canada);

8447 3G ShaneAO: an enhanced adaptive optics and IR imaging system for the Lick Observatory 3meter telescope [8447-125]

R. Kupke, D. Gavel, C. Roskosi, G. Cabak, D. Cowley, D. Dillon, E. L. Gates, R. McGurk, A. Norton, M. Peck, C. Ratliff, M. Reinig, UCO Lick Observatories, Univ. of California, Santa Cruz (United States)

$84473 \mathrm{H} \quad$ Adaptive optical system based on deformable secondary mirror on 1.8-meter telescope [8447-126]

C. Rao, A. Zhang, Institute of Optics and Electronics (China); X. Fan, Y. Guo, Institute of Optics and Electronics (China) and Graduate Univ. of the Chinese Academy of Sciences (China) K. Wei, C. Guan, X. Zhang, Institute of Optics and Electronics (China); C. Li, Institute of Optics and Electronics (China) and Graduate Univ. of the Chinese Academy of Sciences (China) L. Zhou, S. Chen, H. Xian, W. Ma, Y. Cheng, H. Zhou, Y. Zhang, Institute of Optics and Electronics (China)

$844731 \quad$ Adaptive optics for the CHARA array [8447-127]

T. A. ten Brummelaar, L. Sturmann, J. Sturmann, The CHARA Array of Georgia State Univ. (United States); S. T. Ridgway, National Optical Astronomy Observatory (United States); J. D. Monnier, Univ. of Michigan (United States); M. J. Ireland, Macquarie Univ. (Australia) and Australian Astronomical Observatory (Australia); X. Che, Univ. of Michigan (United States); H. A. McAlister, N. H. Turner, CHARA Georgia State Univ. (United States); P. G. Tuthill, Australian Astronomical Observatory (Australia)

8447 3J The 2012 status of the MCAO testbed for the GREGOR solar telescope [8447-128] D. Schmidt, T. Berkefeld, F. Heidecke, Kiepenhever-Institut für Sonnenphysik (Germany)

8447 3K Recent progress on the portable solar adaptive optics [8447-130]

D. Ren, California State Univ. (United States) and Nanjing Institute of Astronomical Optics \& Technology (China); X. Zhang, Nanjing Institute of Astronomical Optics \& Technology (China) and Graduate School of the Chinese Academy of Sciences (China); M. Penn, National Solar Observatory (United States); H. Wang, New Jersey Institute of Technology (United States); J. Dou, Y. Zhu, Nanjing Institute of Astronomical Optics \& Technology (China); L. Rong, X. Wang, Nanjing Institute of Astronomical Optics \& Technology (China) and Graduate School of the Chinese Academy of Sciences (China)

\section{POSTER SESSION: NEW PROPOSED AO SYSTEMS AND CONCEPTS}

8447 3L Designing the METIS adaptive optics system [8447-131]

R. Stuik, Leiden Observatory (Netherlands) and NOVA/ASTRON (Netherlands); S. Hippler, Max-Planck-Institut für Astronomie (Germany); A. Stolte, Argelander Institut für Astronomie, Univ. Bonn (Germany); B. Brandl, Leiden Observatory (Netherlands); F. Molster, NOVA (Netherlands); L. Venema, NOVA/ASTRON (Netherlands); R. Lenzen, Max-Planck-Institut für Astronomie (Germany); E. Pantin, CE Saclay DSM/IRFU/SAp (France); J. Blommaert, Katholieke Univ. Leuven (Belgium); A. Glasse, UK Astronomy Technology Ctr. (United Kingdom); M. Meyer, Institute of Astronomy, ETH Zürich (Switzerland) 
$84473 M \quad$ ERIS adaptive optics system design [8447-132]

E. Marchetti, M. Le Louarn, C. Soenke, E. Fedrigo, P.-Y. Madec, N. Hubin, European Southern Observatory (Germany)

$84473 \mathrm{~N}$ Toward an on-sky ELT-scale sodium LGS wavefront sensing experiment [8447-133] D. Gratadour, G. Rousset, É. Gendron, O. Fauvarque, LESIA - Observatoire de Paris, CNRS, Univ. Paris Diderot, UPMC (France); D. Bonaccini Calia, T. Pfrommer, European Southern Observatory (Germany); R. M. Myers, T. J. Morris, Durham Univ. (United Kingdom)

844730 Optical calibration and testing of the E-ELT M4 adaptive mirror [8447-134] P. Spanò, INAF - Osservatorio Astronomico di Brera (Italy) and NRC Herzberg Institute of Astrophysics (Canada); A. Bianco, INAF - Osservatorio Astronomico di Brera (Italy); R. Briguglio, INAF - Osservatorio Astrofisico di Arcetri (Italy); M. Cecconi, Fundacion Galileo Galilei - INAF (Spain); L. Miglietta, INAF - Osservatorio Astrofisico di Arcetri (Italy); E. Molinari, Fundacion Galileo Galilei - INAF (Spain); G. Pariani, INAF - Osservatorio Astronomico di Brera (Italy); A. Riccardi, INAF - Osservatorio Astrofisico di Arcetri (Italy); M. Riva, D. Tresoldi, INAF - Osservatorio Astronomico di Brera (Italy); M. Xompero, INAF - Osservatorio Astrofisico di Arcetri (Italy); F. Zerbi, INAF - Osservatorio Astronomico di Brera (Italy)

$84473 P \quad$ The Giant Magellan Telescope laser tomography adaptive optics system [8447-135] R. Conan, F. Bennet, Research School of Astronomy and Astrophysics, The Australian National Univ. (Australia); A. H. Bouchez, Giant Magellan Telescope Organization Corp. (United States); M. A. van Dam, Flat Wavefronts (New Zealand); B. Espeland,

W. Gardhouse, C. d'Orgeville, S. Parcell, P. Piatrou, I. Price, F. Rigaut, Research School of Astronomy and Astrophysics, The Australian National Univ. (Australia); G. Trancho, Giant Magellan Telescope Organization Corp. (United States); K. Uhlendorf, Research School of Astronomy and Astrophysics, The Australian National Univ. (Australia)

$84473 Q \quad$ Optical designs of the LGS WFS system for GMT-LTAO [8447-136] M. Wang, Institut National d'Optique (Canada); K. Uhlendorf, Research School of Astronomy and Astrophysics, The Australian National Univ. (Australia); D. Jones, Prime Optics (Australia); P. Côté, F. Châteauneuf, J. Gauvin, Institut National d'Optique (Canada); R. Conan, B. Espeland, Research School of Astronomy and Astrophysics, The Australian National Univ. (Australia)

$84473 R$ Design and predicted performance of the GMT ground-layer adaptive optics mode [8447-137]

P. M. Hinz, G. Brusa, V. Vaitheeswaran, T. McMahon, T. Connors, R. Knox, Steward Observatory, The Univ. of Arizona (United States); A. Bouchez, Giant Magellan Telescope Organization Corp. (United States); M. Montoya, Steward Observatory, The Univ. of Arizona (United States)

844735 The Giant Magellan Telescope phasing system [8447-138]

A. H. Bouchez, Giant Magellan Telescope Organization Corp. (United States); B. A. McLeod, Smithsonian Astrophysical Observatory (United States); D. S. Acton, Ball Aerospace \& Technologies Corp. (United States); S. Kanneganti, Smithsonian Astrophysical Observatory (United States); E. J. Kibblewhite, The Univ. of Chicago (United States); S. A. Shectman, Carnegie Observatories (United States); M. A. van Dam, Flat Wavefronts (New Zealand)

$84473 \mathrm{U}$ Optical design of a Cassegrain mounted AO relay for Imaka [8447-142]

J. Pazder, NRC Herzberg Institute of Astrophysics (Canada) 
$84473 \mathrm{~V}$ A preliminary simulation result of the next-generation wide-field AO at Subaru Telescope [8447-143]

S. Oya, Subaru Telescope, National Astronomical Observatory of Japan (United States); M. Akiyama, Astronomical Institute, Tohoku Univ. (Japan); Y. Hayano, Y. Minowa, I. Iwata, H. Terada, T. Usuda, Subaru Telescope, National Astronomical Observatory of Japan (United States); H. Takami, National Astronomical Observatory of Japan (Japan); T. Nishimura, T. Kodama, N. Takato, D. Tomono, Subaru Telescope, National Astronomical Observatory of Japan (United States); Y. Ono, Astronomical Institute, Tohoku Univ. (Japan)

8447 3W Dimensioning and performances of an AO system for the SALT [8447-144]

L. Catala, South African Astronomical Observatory (South Africa) and Univ. of Cape Town (South Africa); M. Carbillet, Lab. Lagrange, Univ. de Nice Sophia Antipolis, CNRS, Observatoire de la Côte d'Azur (France); L. Jolissaint, aquilAOptics (Switzerland); D. A. H. Buckley, S. M. Crawford, T. Pickering, South African Astronomical Observatory (South Africa)

$84473 X \quad$ System analysis and characterization of the FFREE bench [8447-145] O. Preis, C. Vérinaud, Institut de Planétologie et d'Astrophysique de Grenoble (France); J. Antichi, INAF - Osservatorio Astronomico di Padova (Italy) N. Ventura, Institut de Planétologie et d'Astrophysique de Grenoble (France);

$84473 Y$ Holographic combination of low-resolution Shack-Hartmann sensor and holographybased modal Zernike wavefront sensor [8447-146]

S. Dong, T. Haist, W. Osten, T. Ruppel, O. Sawodny, Univ. Stuttgart (Germany)

844732 Woofer-tweeter adaptive optics in very strong turbulence using a magnetic-liquid deformable mirror [8447-147]

D. Brousseau, COPL, Laval Univ. (Canada); J.-P. Véran, NRC Herzberg Institute of Astrophysics (Canada); S. Thibault, E. F. Borra, S. F.-Boivin, COPL, Laval Univ. (Canada)

844740 Tomographic reconstructor for multi-object adaptive optics using artificial neural networks [8447-148]

D. Guzman, Pontificia Univ. Católica de Chile (Chile); A. T. Mello, Pontificia Univ. Católica de Chile (Chile) and Univ. Federal de Santa Catarina (Brazil); J. Osborn, Pontificia Univ. Católica de Chile (Chile); F. J. De Cos, M. Gómez, Univ. de Oviedo (Spain); T. Butterley, Durham Univ. (United Kingdom); N. David, Pontificia Univ. Católica de Chile (Chile); N. Roqueñi, Univ. de Oviedo (Spain); R. M. Myers, Durham Univ. (United Kingdom);

A. Guesalaga, M. Salas, Pontificia Univ. Católica de Chile (Chile)

$844741 \quad$ Image based deformable mirror control for adaptive optics in satellite telescope [8447-149] N. Miyamura, The Univ. of Tokyo (Japan)

844742 Laboratory demonstration of real time frame selection with Magellan AO [8447-150] J. R. Males, L. M. Close, D. Kopon, Steward Observatory, The Univ. of Arizona (United States); F. Quiros-Pacheco, A. Riccardi, M. Xompero, A. Puglisi, INAF - Osservatorio Astrofisico di Arcetri (Italy); V. Gasho, K. M. Morzinski, K. B. Follette, Steward Observatory, The Univ. of Arizona (United States)

844743 Residual tip-tilt motion of LGS in monostatic scheme [8447-151]

L. A. Bolbasova, V.E. Zuev Institute of Atmospheric Optics (Russian Federation) and Tomsk State Univ. (Russian Federation); V. P. Lukin, V. V. Nosov, V.E. Zuev Institute of Atmospheric Optics (Russian Federation) 
$844744 \quad$ Adaptive optics for laser space debris removal [8447-152]

F. Bennet, R. Conan, C. D'Orgeville, Research School of Astronomy and Astrophysics, The Australian National Univ. (Australia); M. Dawson, EOS Space Systems (Australia); N. Paulin, I. Price, F. Rigaut, Research School of Astronomy and Astrophysics, The Australian National Univ. (Australia); I. Ritchie, C. Smith, EOS Space Systems (Australia); K. Uhlendorf, Research School of Astronomy and Astrophysics, The Australian National Univ. (Australia)

844745 Concepts, laboratory, and telescope test results of the plenoptic camera as a wavefront sensor [8447-153]

L. F. Rodríguez-Ramos, I. Montilla, Instituto de Astrofísica de Canarias (Spain);

J. J. Fernández-Valdivia, J. L. Trujillo-Sevilla, J. M. Rodríguez-Ramos, Univ. de La Laguna (Spain)

POSTER SESSION: NEW OBSERVATIONS MADE WITH AO, USE, AND CAPABILITIES OF NEW SYSTEMS

844746 An updated 37-element low-order solar adaptive optics system for 1-m new vacuum solar telescope at Full-Shine Lake Solar Observatory [8447-154]

C. Rao, L. Zhu, N. Gu, X. Rao, Institute of Optics and Electronics (China); L. Zhang, Institute of Optics and Electronics (China) and Graduate School of Chinese Academy of Sciences (China); C. Guan, D. Chen, S. Chen, C. Wang, Institute of Optics and Electronics (China);

J. Lin, Z. Liu, Yunnan Astronomical Observatory (China)

844747 Performances analysis of SINFONI with the laser guide star facility [8447-155]

J. P. O'Neal, F. Y. J. Gonte, European Southern Observatory (Chile)

$84474 \mathrm{~A} \quad$ Image quality analyzer [8447-158]

V. P. Lukin, N. N. Botugina, O. N. Emaleev, L. V. Antoshkin, P. A. Konyaev, V.E. Zuev Institute of Atmospheric Optics (Russian Federation)

8447 4D Solar adaptive optics at the Hida Observatory: latest achievements of current system and design of new system [8447-162]

N. Miura, J. Miyazaki, S. Kuwamura, Kitami Institute of Technology (Japan); N. Baba, Hokkaido Univ. (Japan); Y. Hanaoka, National Astronomical Observatory of Japan (Japan); M. Yamaguchi, S. Ueno, Y. Nakatani, S. Nagata, R. Kitai, K. Ichimoto, Kyoto Univ. (Japan); H. Takami, Subaru Telescope, National Astronomical Observatory of Japan (United States)

8447 4E Optical calibration of capacitive sensors for AO: strategy and preliminary results [8447-163] R. Briguglio, M. Xompero, A. Riccardi, INAF - Osservatorio Astrofisico di Arcetri (Italy); R. Biasi, M. Andrighettoni, MICROGATE srl (Italy)

\section{POSTER SESSION: LASER GUIDE STAR SYSTEMS}

$84474 \mathrm{~F} \quad$ Keck I laser guide star adaptive optics system [8447-164]

J. C. Y. Chin, P. Wizinowich, R. Campbell, L. Chock, A. Cooper, E. James, J. Lyke, J. Mastromarino, O. Martin, D. Medeiros, D. Morrison, C. Neyman, S. Panteleev, T. Stalcup, P. Tucker, E. Wetherell, W. M. Keck Observatory (United States); M. van Dam, Flat Wavefronts (New Zealand) 
$84474 \mathrm{H} \quad$ Performance of the SOAR adaptive module with UV Rayleigh guide star [8447-166] A. Tokovinin, R. Tighe, P. Schurter, R. Cantarutti, N. van der Bliek, M. Martinez, E. Mondaca, S. Heathcote, Cerro Tololo Inter-American Observatory (Chile)

84474 Testing and integrating the laser system of ARGOS: the ground layer adaptive optics for LBT [8447-167]

C. Loose, S. Rabien, L. Barl, Max-Planck-Institut für extraterrestrische Physik (Germany); J. Borelli, Max-Planck-Institut für Astronomie (Germany); M. Deysenroth, Max-Planck-Institu† für extraterrestrische Physik (Germany); W. Gaessler, Max-Planck-Institut für Astronomie (Germany); H. Gemperlein, M. Honsberg, Max-Planck-Institut für extraterrestrische Physik (Germany); M. Kulas, Max-Planck-Institut für Astronomie (Germany); R. Lederer, W. Raab, Max-Planck-Institut für extraterrestrische Physik (Germany); G. Rahmer, Large Binocular Telescope (United States); J. Ziegleder, Max-Planck-Institut für extraterrestrische Physik (Germany)

$84474 J \quad$ Vibration control for the ARGOS laser launch path [8447-168]

D. Peter, W. Gässler, J. Borelli, Max-Planck-Institut für Astronomie (Germany); L. Barl, S. Rabien, Max-Planck-Institut für extraterrestrische Physik (Germany)

8447 4K A sodium guide star adaptive optics system for the 1.8 meter telescope [8447-169] K. Wei, C. Rao, Institute of Optics and Electronics (China); Y. Bo, Technical Institute of Physics and Chemistry (China); C. Li, M. Li, X. Zhang, A. Zhang, C. Guan, L. Zhou, S. Chen, X. Hao, W. Ma, Y. Zhang, Institute of Optics and Electronics (China)

$84474 \mathrm{~L}$ Investigations of long pulse sodium laser guide stars [8447-170] R. Rampy, D. Gavel, Univ. of California, Santa Cruz (United States); S. Rochester, Rochester Scientific LLC (United States); R. Holzlöhner, European Southern Observatory (Germany)

$84474 \mathrm{M}$ Improving stability, robustness, and performance of laser systems [8447-171] A. Guesalaga, Univ. Católica de Chile (Chile); B. Neichel, M. Boccas, C. D'Orgeville, F. Rigaut, Gemini Observatory Southern Operations Ctr. (Chile); D. Guzman, Univ. Católica de Chile (Chile); J. Anguita, Univ. de Los Andes (Chile)

$84474 \mathrm{~N}$ Design, analysis, and testing of the optical tube assemblies for the ESO VLT four laser guide star facility [8447-172]

R. Henselmans, D. Nijkerk, M. Lemmen, N. Rijnveld, F. Kamphues, TNO (Netherlands)

844740 Design of the multiple Laser Guide Stars wavefront sensor prototype for the EELT [8447-173] M. Lombini, I. Foppiani, INAF - Osservatorio Astronomico di Bologna (Italy); L. Schreiber, INAF - Osservatorio Astronomico di Padova (Italy); E. Diolaiti, G. Bregoli, INAF - Osservatorio Astronomico di Bologna (Italy); G. Cosentino, Univ. degli Studi di Bologna (Italy)

8447 4P Dynamical refocusing laser guide stars with membrane mirrors [8447-175] S. Rabien, J. Ziegleder, Max-Planck-Institut für extraterrestrische Physik (Germany)

$84474 Q$ Science readiness of the Gemini MCAO system: GeMS [8447-176] B. Neichel, Gemini Observatory (Chile); F. Rigaut, Research School of Astronomy and Astrophysics, The Australian National Univ. (Australia); A. Serio, G. Arriagada, M. Boccas, Gemini Observatory (Chile); C. d'Orgeville, Research School of Astronomy and Astrophysics, The Australian National Univ. (Australia); V. Fesquet, C. Trujillo, W. N. Rambold, R. L. Galvez, G. Gausachs, T. B. Vucina, V. Montes, C. Urrutia, C. Moreno, S. J. Diggs, C. Araya, J. Lührs, Gemini Observatory (Chile); G. Trancho, M. Bec, Giant Magellan 
Telescope Organization Corp. (United States); C. Marchant, F. Collao, E. R. Carrasco, M. L. Edwards, P. Pessev, A. Lopez, H. Diaz, Gemini Observatory (Chile)

$84474 R \quad$ Recent developments in aircraft protection systems for laser guide star operations [8447-177]

P. J. Stomski, Jr., W. M. Keck Observatory (United States); T. W. Murphy, Univ. of California, San Diego (United States); R. Campbell, W. M. Keck Observatory (United States)

8447 4S A decade of operations with the laser traffic control system: paradigm shift and implied development directions [8447-261]

D. Summers, W. M. Keck Observatory (United States); D. Abrams, J. Skvarč, Isaac Newton Group of Telescopes (Spain); P. Amico, H. Kuntschner, European Southern Observatory (Germany)

\section{POSTER SESSION: PATHFINDERS TO ENABLE AO ON ELTS AND NEW AO CONCEPTS}

$84474 \mathrm{~V}$ Intelligent vibration control of ELTs and large AO hardware [8447-180] J.-U. Pott, M. Kürster, J. Trowitzsch, J. Borelli, R.-R. Rohloff, T. Herbst, Max-Planck-Institut für Astronomie (Germany); M. Böhm, A. Keck, Max-Planck-Institut für Astronomie (Germany) and Univ. Stuttgart (Germany); T. Ruppel, O. Sawodny, Univ. Stuttgart (Germany)

8447 WW Development of new concepts to minimize the impact of fast telescope vibrations seen by the E-ELT/MICADO wavefront sensors [8447-181]

A. Keck, Univ. Stuttgart (Germany); J.-U. Pott, Max-Planck-Institut für Astronomie (Germany); T. Ruppel, O. Sawodny, Univ. Stuttgart (Germany)

$84474 X \quad$ A NGSs based WFS for the E-ELT and the VLT [8447-182] J. Farinato, R. Ragazzoni, D. Magrin, V. Viotto, INAF - Osservatorio Astronomico di Padova (Italy); M. Bergomi, INAF - Osservatorio Astronomico di Padova (Italy) and Univ. degli Studi di Padova (Italy); A. Brunelli, M. Dima, INAF - Osservatorio Astronomico di Padova (Italy); L. Marafatto, Univ. degli Studi di Padova (Italy)

8447 4Y DRAGON: a wide-field multipurpose real time adaptive optics test bench [8447-183] A. P. Reeves, R. M. Myers, T. J. Morris, A. G. Basden, N. A. Bharmal, S. Rolt, D. G. Bramall, N. A. Dipper, E. J. Younger, Durham Univ. (United Kingdom)

$84474 Z$ Toward an experimental validation of new AO concepts for future E-ELT instrumentation [8447-184]

K. El Hadi, Aix-Marseille Univ., CNRS, Lab. d'Astrophysique de Marseille (France); T. Fusco, ONERA (France) and Aix-Marseille Univ., CNRS, Lab. d'Astrophysique de Marseille (France); B. Le Roux, Aix-Marseille Univ., CNRS, Lab. d'Astrophysique de Marseille (France)

844750 The HIA MCAO laboratory bench [8447-185] J.-P. Véran, E. McWeigh, D. Andersen, C. Correia, G. Herriot, J. Pazder, NRC Herzberg Institute of Astrophysics (Canada)

844751 A test bench for ARGOS: integration of sub-systems and validation of the wavefront sensing [8447-186]

G. Orban de Xivry, S. Rabien, Max-Planck-Institut für extraterrestrische Physik (Germany) 
844752 A prototype phasing camera for the Giant Magellan Telescope [8447-187]

S. Kanneganti, B. A. McLeod, M. P. Ordway, J. B. Roll, Jr., Harvard-Smithsonian Ctr. for Astrophysics (United States); S. A. Shectman, A. H. Bouchez, Giant Magellan Telescope Organization Corp. (United States); J. Codona, Steward Observatory, The Univ. of Arizona (United States); R. Eng, T. M. Gauron, F. Handte, T. J. Norton, P. Streechon, D. Weaver, Harvard-Smithsonian Ctr. for Astrophysics (United States)

844753 Final opto-mechanical design of Raven, a MOAO science demonstrator for Subaru [8447-188]

O. Lardière, R. Nash, J.-P. Markes, Univ. of Victoria (Canada); D. Andersen, NRC Herzberg Institute of Astrophysics (Canada); C. Bradley, C. Blain, R. Desmarais, D. Gamroth, M. Ito, K. Jackson, P. Lach, L. Pham, Univ. of Victoria (Canada)

844754 Design and test results of the calibration unit for the MOAO demonstrator RAVEN [8447-189] J-F. Lavigne, ABB Bomem (Canada); F. Lamontagne, G. Anctil, M. Wang, M. Tremblay, INO (Canada); O. Lardière, R. Nash, Univ. of Victoria (Canada); D. Andersen, NRC Herzberg Institute of Astrophysics (Canada); M. Savard, P. Côté, INO (Canada); C. H. Bradley, Univ. of Victoria (Canada); F. Châteauneuf, INO (Canada)

844755 MOAO test bench in Tohoku University [8447-190]

M. Akiyama, Y. Ono, Tohoku Univ. (Japan); S. Oya, Subaru Telescope, National

Astronomical Observatory of Japan (United States); K. Hane, T. WU, Tohoku Univ. (Japan)

\section{Part Four}

POSTER SESSION: CHARACTERIZATION, MEASUREMENT AND MODELING OF THE DISTURBANCES FACED BY AO

844756 Atmospheric coherent turbulence [8447-191]

V. V. Nosov, P. G. Kovadlo, V. P. Lukin, A. V. Torgaev, V.E. Zuev Institute of Atmospheric Optics (Russian Federation)

844757 Impact of $\mathrm{Cn}^{2}$ profile on tomographic reconstruction performance: application to E-ELT wide field AO systems [8447-192]

A. Costille, Institut de Planétologie et d'Astrophysique de Grenoble (France); T. Fusco, ONERA (France)

844759 Accurate measurement of $\mathrm{Cn}^{2}$ profile with Shack-Hartmann data [8447-194]

J. Voyez, C. Robert, V. Michau, J.-M. Conan, T. Fusco, ONERA (France)

8447 5A MOSE: a feasibility study for optical turbulence forecast with the Meso-Nh mesoscale model to support AO facilities at ESO sites (Paranal and Armazones) [8447-195]

E. Masciadri, F. Lascaux, INAF - Osservatorio Astrofisico di Arcetri (Italy)

8447 5B MOSE: zooming on the Meso-NH mesoscale model performances at the surface layer at ESO sites (Paranal and Armazones) [8447-196]

F. Lascaux, E. Masciadri, INAF - Osservatorio Astrofisico di Arcetri (Italy) 
8447 5D Simulations of adaptive optics systems for the E-ELT [8447-199] M. Le Louarn, R. Clare, European Southern Observatory (Germany); C. Béchet, M. Tallon, CRAL, Observatoire de Lyon (France)

8447 5E A study of MOAO behind GLAO for EAGLE [8447-200]

A. Basden, N. A. Bharmal, T. Butterley, N. Dipper, T. Morris, R. Myers, A. Reeves, Durham Univ. (United Kingdom)

$84475 \mathrm{~F}$ Tomographic wavefront error estimation and measurement for Raven, a multi-object adaptive optics demonstrator [8447-201]

K. Jackson, Univ. of Victoria Mechanical Engineering (Canada); C. Correia, NRC Herzberg Institute of Astrophysics (Canada); O. Lardière, Univ. of Victoria Mechanical Engineering (Canada); D. Andersen, NRC Herzberg Institute of Astrophysics (Canada); C. Bradley, Univ. of Victoria Mechanical Engineering (Canada)

8447 5G GMT AO system requirements and error budgets in the preliminary design phase [8447-202] G. Trancho, Giant Magellan Telescope Organization Corp. (United States); B. Espeland, Research School of Astronomy and Astrophysics, The Australian National Univ. (Australia); A. Bouchez, Giant Magellan Telescope Organization Corp. (United States); R. Conan, Research School of Astronomy and Astrophysics, The Australian National Univ. (Australia); P. Hinz, Steward Observatory (United States); M. van Dam, Flat Wavefronts (New Zealand)

$84475 \mathrm{H} \quad$ Multi-conjugate AO for the European Solar Telescope [8447-203]

I. Montilla, Instituto de Astrofísica de Canarias (Spain); C. Béchet, Ctr. de Recherche Astronomique de Lyon (France); M. Le Louarn, European Southern Observatory (Germany); M. Tallon, Ctr. de Recherche Astronomique de Lyon (France); J. Sánchez-Capuchino, M. Collados Vera, Instituto de Astrofísica de Canarias (Spain)

844751 Optimal projection of reconstructed layers onto deformable mirrors with fractal iterative method for AO tomography [8447-204]

E. Brunner, Ctr. de Recherche Astrophysique de Lyon, Observatoire de Lyon (France) and Technische Univ. München (Germany); C. Béchet, M. Tallon, Ctr. de Recherche Astrophysique de Lyon, Observatoire de Lyon (France)

$84475 \mathrm{~J} \quad$ Ground layer adaptive optics system simulation for the $2.5 \mathrm{~m}$ telescope in Dome A [8447-205]

P. Jia, Nanjing Univ. (China) and Nanjing Institute of Astronomical Optics \& Technology (China); S. Zhang, Nanjing Institute of Astronomical Optics \& Technology (China)

$84475 \mathrm{~K}$ Adaptive optics performance simulation on the basis of MASS/DIMM data obtained on Mt. Shatdzhatmaz in 2009-2011 [8447-206]

B. S. Safonov, Moscow MV Lomonosov State Univ., Sternberg Astronomical Institute (Russian Federation)

$84475 \mathrm{~L} \quad$ Performance simulation of the ERIS pyramid wavefront sensor module in the VLT adaptive optics facility [8447-207]

F. Quirós-Pacheco, G. Agapito, A. Riccardi, S. Esposito, INAF - Osservatorio Astrofisico di Arcetri (Italy); M. Le Louarn, E. Marchetti, European Southern Observatory (Germany) 
$84475 \mathrm{M}$ Calibration strategy of the pyramid wavefront sensor module of ERIS with the VLT deformable secondary mirror [8447-208]

A. Riccardi, R. Briguglio, E. Pinna, G. Agapito, F. Quiros-Pacheco, S. Esposito, INAF Osservatorio Astrofisico di Arcetri (Italy)

$84475 \mathrm{~N}$ Identification and calibration of the interaction matrix parameters for AO and MCAO systems [8447-209]

B. Neichel, Gemini Observatory (Chile); A. Parisot, C. Petit, T. Fusco, ONERA (France);

F. Rigaut, Gemini Observatory (Chile)

844750 Analysis techniques for adaptively controlled segmented mirror arrays [8447-212] G. J. Michels, V. L. Genberg, Sigmadyne, Inc. (United States)

$84475 R \quad$ GPUs for adaptive optics: simulations and real-time control [8447-215]

D. Gratadour, A. Sevin, J. Brulé, É. Gendron, G. Rousset, LESIA, Observatoire de Paris, CNRS, Univ. Paris Diderot, UPMC (France)

$84475 S$ Efficient iterative atmospheric tomography reconstruction from LGS and additional tip/tilt measurements [8447-216]

M. Rosensteiner, MathConsult GmbH (Austria); R. Ramlau, Johannes Kepler Univ. of Linz (Austria)

\section{POSTER SESSION: POST-PROCESSING OF AO DATA}

8447 5T New results on a Cn² profiler for GeMS [8447-217]

A. Cortes, Pontificia Univ. Católica de Chile (Chile); B. Neichel, Gemini Observatory (Chile); A. Guesalaga, J. Osborn, Pontificia Univ. Católica de Chile (Chile); F. Rigaut, Research School of Astronomy and Astrophysics, The Australian National Univ. (Australia); D. Guzman, Pontificia Univ. Católica de Chile (Chile)

$84475 \mathrm{U}$ What can be retrieved from adaptive optics real-time data? [8447-219] J. Kolb, N. Muller, E. Aller-Carpentier, European Southern Observatory (Germany); P. Andrade, Faculdade de Engenharia da Univ. do Porto (Portugal); J. Girard, European Southern Observatory (Chile)

$84475 \mathrm{~V}$ Developing a new software package for PSF estimation and fitting of adaptive optics images [8447-220]

L. Schreiber, INAF, Astronomical Observatory of Padova (Italy); E. Diolaiti, INAF Osservatorio Astronomico di Bologna (Italy); A. Sollima, INAF - Osservatorio Astronomico di Padova (Italy); C. Arcidiacono, M. Bellazzini, P. Ciliegi, INAF - Osservatorio Astronomico di Bologna (Italy); R. Falomo, INAF - Osservatorio Astronomico di Padova (Italy); I. Foppiani, INAF - Osservatorio Astronomico di Bologna (Italy); L. Greggio, INAF - Osservatorio Astronomico di Padova (Italy); B. Lanzoni, Univ. degli Studi di Bologna (Italy); M. Lombini, P. Montegriffo, INAF - Osservatorio Astronomico di Bologna (Italy); E. Dalessandro,

D. Massari, Univ. degli Studi di Bologna (Italy)

8447 5W PSF reconstruction for MUSE in wide field mode [8447-221]

R. Villecroze, CRAL, Observatoire de Lyon (France) and ONERA (France); T. Fusco, ONERA (France); R. Bacon, CRAL, Observatoire de Lyon (France); P.-Y. Madec, European Southern Observatory (Germany) 
$84475 X \quad$ Estimation of errors on the PSF reconstruction process for myopic deconvolution [8447-222] J. Exposito, D. Gratadour, Y. Clénet, G. Rousset, LESIA, Observatoire de Paris, CNRS, UPMC, Univ. Paris-Diderot (France); L. Mugnier, ONERA (France)

$84475 Y \quad$ Statistical moments of the Strehl ratio [8447-223]

N. Yaitskova, M. Esselborn, European Southern Observatory (Germany); S. Gladysz, Fraunhofer Institute of Optronics, System Technologies and Image Exploitation (Germany)

$84475 Z$ Experimental validation of optimization concepts for focal-plane image processing with adaptive optics [8447-224]

V. Korkiakoski, C. U. Keller, Leiden Observatory (Netherlands); N. Doelman, TNO Science and Industry (Netherlands); R. Fraanje, R. Andrei, M. Verhaegen, Delft Ctr. for Systems and Control (Netherlands)

844760 Preserving the photometric integrity of companions in high-contrast imaging observations using locally optimized combination of images [8447-225]

J. Maire, Dunlap Institute for Astronomy and Astrophysics, Univ. of Toronto (Canada);

J. Gagné, D. Lafrenière, R. Doyon, Univ. de Montréal (Canada); J. R. Graham, Dunlap Institute for Astronomy and Astrophysics, Univ. of Toronto (Canada); J.-P. Véran, NRC Herzberg Institute of Astrophysics (Canada); L. A. Poyneer, Lawrence Livermore National Lab. (United States)

\section{POSTER SESSION: WAVEFRONT CORRECTION}

844761 Specifications and design of the E-ELT M4 adaptive unit [8447-226]

E. Vernet, M. Cayrel, N. Hubin, M. Mueller, European Southern Observatory (Germany);

R. Biasi, Microgate S.r.I. (Italy); D. Gallieni, M. Tintori, A.D.S. International S.r.l. (Italy)

844762 Numerical modeling and simulation studies for the M4 adaptive mirror of the E-ELT [8447-227]

M. Carbillet, Lab. Lagrange, Univ. de Nice Sophia-Antipolis, CNRS, Observatoire de la Côte d'Azur (France); A. Riccardi, M. Xompero, INAF - Osservatorio Astrofisico di Arcetri (Italy)

844763 LBT adaptive secondary mirrors: chopping procedures and optical calibration on the test bench [8447-228]

R. Briguglio, M. Xompero, A. Riccardi, INAF - Osservatorio Astrofisico di Arcetri (Italy)

$844764 \quad$ Novel unimorph adaptive mirrors for astronomy applications [8447-229]

P. Rausch, S. Verpoort, U. Wittrock, Münster Univ. of Applied Sciences (Germany)

844765 Deformable mirrors for open-loop adaptive optics [8447-230]

A. Kellerer, F. Vidal, E. Gendron, Z. Hubert, D. Perret, G. Rousset, LESIA, Observatoire de Paris, CNRS, UPMC, Univ. Paris Diderot (France)

844766 Conceptual design for a deformable mirror for use with $\mathbf{x}$-ray sources [8447-232] M. Hart, J. Codona, R. Codona, Steward Observatory, The Univ. of Arizona (United States); S. M. Ammons, B. A. Macintosh, T. McCarville, T. Pardini, M. Pivovaroff, L. Poyneer, Lawrence Livermore National Lab. (United States) 
844767 Reference design of deformable mirror electronics for ELT systems [8447-233]

K. Caputa, G. Herriot, National Research Council Canada (Canada); J. Niebergal,

A. Zielinski, Univ. of Victoria (Canada)

844768 Open-loop control of SCEXAO's MEMS deformable mirror using the Fast Iterative Algorithm: speckle control performances [8447-234]

C. Blain, Univ. of Victoria (Canada); O. Guyon, F. Martinache, Subaru Telescope, National Astronomical Observatory of Japan (United States); C. Bradley, C. Clergeon, Univ. of Victoria (Canada)

844769 Ziegler-Nichols frequency response method for high-order adaptive optics system of the Advanced Technology Solar Telescope [8447-235]

J. Curamen, M3 Engineering and Technology (United States) and National Solar Observatory (United States); L. Johnson, T. Rimmele, National Solar Observatory (United States)

\section{POSTER SESSION: WAVEFRONT SENSING}

8447 6A An overview of AONGC and the ESO adaptive optics wave front sensing camera [8447-237]

J. Reyes, M. Downing, R. Conzelmann, L. Mehrgan, J. Stegmeier, M. Todorovic, European Southern Observatory (Germany); I. Molina-Conde, Univ. of Malaga (Spain)

$84476 B \quad$ Laboratory characterization of the ARGOS laser wavefront sensor [8447-238] M. Bonaglia, L. Busoni, L. Carbonaro, F. Quiròs Pacheco, M. Xompero, S. Esposito, INAF Osservatorio Astrofisico di Arcetri (Italy); G. Orban de Xivry, S. Rabien, Max-Planck-Institut für extraterrestrische Physik (Germany)

8447 6D Characterization of an off-the-shelf detector for high-order wavefront sensing in solar adaptive optics [8447-240]

L. C. Johnson, K. Richards, F. Wöger, S. Barden, T. Rimmele, National Solar Observatory (United States)

8447 6E Development of adaptive optics elements for solar telescope [8447-241]

V. P. Lukin, V.E. Zuev Institute of Atmospheric Optics (Russian Federation); V. M. Grigor'ev, Institute of Solar-Terrestrial Physics (Russian Federation); L. V. Antoshkin, N. N. Botugina, V.E. Zuev Institute of Atmospheric Optics (Russian Federation); P. G. Kovadlo, Institute of Solar-Terrestrial Physics (Russian Federation); P. A. Konyaev, E. A. Kopulov, V.E. Zuev Institute of Atmospheric Optics (Russian Federation); V. I. Skomorovsky, V. D. Trifonov,

S. A. Chuprakov, Institute of Solar-Terrestrial Physics (Russian Federation)

8447 6F Aligning a more than 100 degrees of freedom wavefront sensor [8447-242] L. Marafatto, Univ. degli Studi di Padova (Italy); M. Bergomi, Univ. degli Studi di Padova (Italy) and INAF - Astronomical Observatory of Padova (Italy); A. Brunelli, M. Dima, J. Farinato, G. Farisato, L. Lessio, D. Magrin, R. Ragazzoni, V. Viotto, INAF - Osservatorio Astronomico di Padova (Italy); T. Bertram, P. Bizenberger, M. Brangier, F. Briegel, A. Conrad, F. De Bonis, T. Herbst, R. Hofferbert, F. Kittmann, M. Kürster, D. Meschke, L. Mohr, R. R. Rohloff, Max-Planck-Institut für Astronomie (Germany) 
8447 6H The LINC-NIRVANA high layer wavefront sensor laboratory experiment: progress report [8447-244]

X. Zhang, Max-Planck-Institut für Astronomie (Germany) and Institute of Optics and Electronics (China) and Graduate School of Chinese Academy of Sciences (China); A. R. Conrad, D. Meschke, T. Bertram, T. M. Herbst, Max-Planck-Institut für Astronomie (Germany); C. Arcidiacono, INAF - Osservatorio Astronomico di Bologna (Italy) and INAF Arcetri Astrophysical Observatory (Italy); P. Bizenberger, W. Gaessler, Max-Planck-Institut für Astronomie (Germany); L. Schreiber, R. Ragazzoni, INAF - Astronomical Observatory of Padova (Italy); M. Kuerster, F. De Bonis, L. Mohr, Max-Planck-Institut für Astronomie (Germany); J. Farinato, INAF - Osservatorio Astronomico di Padova (Italy); E. Diolaiti, INAF Osservatorio Astronomico di Bologna (Italy); H.-W. Rix, Max-Planck-Institut für Astronomie (Germany); C. Rao, Institute of Optics and Electronics (China); F. Briegel, F. Kittmann, J. Berwein, J. Trowitzsch, M. Brangier, Max-Planck-Institut für Astronomie (Germany)

$844761 \quad$ Pyramidal wavefront sensor using diffractive lenses [8447-245]

M. P. Cagigal, J. E. Oti, M. A. Cagigas, P. J. Valle, Univ. de Cantabria (Spain)

$84476 \mathrm{~K}$ Testing the pyramid wavefront sensor without modulation used in the closed-loop adaptive optics system [8447-247]

S. Wang, C. Rao, A. Zhang, X. Zhang, K. Wei, Y. Tian, Z. Liao, C. Zhang, H. Xian, X. Zhang,

L. Wei, Institute of Optics and Electronics (China)

$84476 \mathrm{~L} \quad$ Influence of atmospheric turbulence on the Zernike phase contrast method and the first steps towards the phasing of segmented deformable mirrors [8447-248]

I. Surdej, E. Romnée, R. Bastaits, A. Preumont, Univ. Libre de Bruxelles (Belgium);

N. Yaitskova, L. Noethe, European Southern Observatory (Germany)

$84476 \mathrm{M}$ Applications of absolute surface metrology by transverse shifting [8447-249]

E. E. Bloemhof, National Science Foundation (United States)

8447 6N Temporal analysis of aliasing in Shack-Hartmann wave-front sensing [8447-250]

E. Gendron, G. Rousset, LESIA, Observatoire de Paris, CNRS, UPMC, Univ. Paris Didero

(France)

$84476 \mathrm{P}$ Theory and application of differential OTF (dOTF) wavefront sensing [8447-252]

J. L. Codona, Steward Observatory, The Univ. of Arizona (United States)

$84476 Q \quad$ Application of dOTF wavefront sensing to 3D aberration measurement in an optical system [8447-253]

M. Hart, J. L. Codona, Steward Observatory, The Univ. of Arizona (United States)

8447 6R Experimental evaluation of differential OTF (dOTF) wavefront sensing [8447-254]

J. L. Codona, Steward Observatory, The Univ. of Arizona (United States); N. Doble, New

England College of Optometry (United States)

844765 Focal plane wavefront sensing and control for ground-based imaging [8447-255]

D. Savransky, B. A. Macintosh, Lawrence Livermore National Lab. (United States);

S. J. Thomas, Gemini Observatory (Chile); L. A. Poyneer, D. W. Palmer, Lawrence Livermore National Lab. (United States); R. J. De Rosa, Univ. of Exeter (United Kingdom); M. Hartung, Gemini Observatory (Chile) 
8447 6T Bilinear solution to the phase diversity problem for extended objects based on the Born approximation [8447-256]

R. M. Andrei, R. Fraanje, M. Verhaegen, Delft Ctr. for Systems and Control (Netherlands); V. A. Korkiakoski, C. U. Keller, Leiden Observatory (Netherlands); N. Doelman, TNO Science and Industry (Netherlands)

$84476 \mathrm{U}$ Linear analytical solution to the phase diversity problem for extended objects based on the Born approximation [8447-257]

R. M. Andrei, C. S. Smith, R. Fraanje, M. Verhaegen, Delft Ctr. for Systems and Control (Netherlands); V. A. Korkiakoski, C. U. Keller, Leiden Observatory (Netherlands); N. Doelman, TNO Science and Industry (Netherlands)

$84476 \mathrm{~V} \quad$ Fast phase diversity wavefront sensing using object independent metrics [8447-258] C. S. Smith, A. J. den Dekker, R. Andrei, R. Fraanje, M. Verhaegen, Delft Univ. of Technology (Netherlands)

8447 6W A first order wavefront estimation algorithm for P1640 calibrator [8447-259]

C. Zhai, G. Vasisht, M. Shao, T. Lockhart, E. Cady, Jet Propulsion Lab. (United States);

B. Oppenheimer, American Museum of Natural History (United States); R. Burruss, J. Roberts, Jet Propulsion Lab. (United States); C. Beichman, California Institute of Technology (United States); D. Brenner, American Museum of Natural History (United States); J. Crepp, R. Dekany, S. Hinkley, L. Hillenbrand, California Institute of Technology (United States); E. R. Ligon, Jet Propulsion Lab. (United States); I. Parry, Cambridge Univ. (United Kingdom); L. Pueyo, Johns Hopkins Univ. (United States); E. Rice, American Museum of Natural History (United States); L. C. Roberts, Jr., Jet Propulsion Lab. (United States); A. Sivaramakrishnan, Space Telescope Science Institute (United States); R. Soummer, Johns Hopkins Univ. (United States); F. Vescelus, K. Wallace, Jet Propulsion Lab. (United States); N. Zimmerman, Max Planck Institute for Astronomy (Germany)

8447 6X MCAO: Wavefront sensing only as a tool for high precision photometry? [8447-260] V. Viotto, R. Ragazzoni, INAF - Osservatorio Astronomico di Padova (Italy); M. Bergomi, INAF - Osservatorio Astronomico di Padova (Italy) and Univ. degli Studi di Padova (Italy); A. Brunelli, M. Dima, J. Farinato, D. Magrin, INAF - Osservatorio Astronomico di Padova (Italy); L. Marafatto, V. Nascimbeni, G. Piotto, Univ. degli Studi di Padova (Italy)

Author Index 
Proc. of SPIE Vol. $8447844701-34$

Downloaded From: https://www.spiedigitallibrary.org/conference-proceedings-of-spie on 26 Apr 2023 Terms of Use: https://www.spiedigitallibrary.org/terms-of-use 


\section{Conference Committee}

Symposium Chairs

Mark M. Casali, European Southern Observatory (Germany)

Kathryn A. Flanagan, Space Telescope Science Institute

(United States)

Symposium Cochairs

Gillian S. Wright, UK Astronomy Technology Centre (United Kingdom)

Luc Smiard, National Research Council Canada (Canada)

Conference Chairs

Brent L. Ellerbroek, Thirty Meter Telescope Observatory Corporation (United States)

Enrico Marchetti, European Southern Observatory (Germany)

Jean-Pierre Véran, National Research Council Canada (Canada)

Conference Program Committee

Laird M. Close, The University of Arizona (United States)

Rodolphe Conan, The Australian National University (Australia)

Emiliano Diolaiti, INAF - Osservatorio Astronomico di Bologna (Italy)

Thierry Fusco, ONERA (France)

Donald T. Gavel, University of California, Santa Cruz (United States)

Michael Lloyd-Hart, The University of Arizona (United States)

Yutaka Hayano, Subaru Telescope, National Astronomical

Observatory of Japan (United States)

Glen Herriot, National Research Council Canada (Canada)

Norbert Hubin, European Southern Observatory (Germany)

Markus Kasper, European Southern Observatory (Germany)

Caroline Kulcsár, Université Paris 13 (France)

Anne-Marie Lagrange, Laboratoire d'Astrophysique de l'Observatoire de Grenoble (France)

Bruce A. Macintosh, Lawrence Livermore National Laboratory (United States)

Pierre-Yves Madec, European Southern Observatory (Germany)

Richard M. Myers, Durham University (United Kingdom)

Armando Riccardi, INAF - Osservatorio Astrofisico di Arcetri (Italy)

François Rigaut, The Australian National University (Australia) 
Andrei A. Tokovinin, National Optical Astronomy Observatory (United States)

Mitchell Troy, Jet Propulsion Laboratory (United States)

Peter L. Wizinowich, W. M. Keck Observatory (United States)

\section{Session Chairs}

1 Project Status I

Peter L. Wizinowich, W. M. Keck Observatory (United States)

2 Wavefront Correctors I

Armando Riccardi, INAF - Osservatorio Astrofisico di Arcetri (Italy)

3 Quantitative Astronomy and Science with AO I

Markus Kasper, European Southern Observatory (Germany)

$4 \quad$ Laser Systems

François Rigaut, The Australian National University (Australia)

5 Project Status II

Glen Herriot, National Research Council Canada (Canada)

6 Quantitative Astronomy and Science with AO II

Markus Kasper, European Southern Observatory (Germany)

7 Wavefront Sensing I

Enrico Marchetti, European Southern Observatory (Germany)

8 Project Status III

Norbert Hubin, European Southern Observatory (Germany)

9 Advances in AO Control I

Donald T. Gavel, University of California, Santa Cruz (United States)

10 Wavefront Sensing II

Emiliano Diolaiti, INAF - Osservatorio Astronomico di Bologna (Italy)

11 AO Disturbances Modeling and Characterization I

Enrico Marchetti, European Southern Observatory (Germany)

12 AO Disturbances Modeling and Characterization II

Enrico Marchetti, European Southern Observatory (Germany)

13 Project Status IV

Jean-Pierre Véran, National Research Council Canada (Canada) 
14 AO for ELTs

Richard M. Myers, Durham University (United Kingdom)

15 Wavefront Sensing III

Brent L. Ellerbroek, Thirty Meter Telescope Observatory Corporation (United States)

16 Laser System Tests

Brent L. Ellerbroek, Thirty Meter Telescope Observatory Corporation (United States)

17 Advances in AO Control II

Caroline Kulcsár, Université Paris 13 (France)

18 Extreme $\mathrm{AO}$

Bruce A. Macintosh, Lawrence Livermore National Laboratory (United States)

19 AO Modeling, Analysis, and Simulations

Rodolphe Conan, The Australian National University (Australia)

20 Postprocessing of AO Data

Laird M. Close, The University of Arizona (United States)

21 Advances in AO Control III

Michael Lloyd-Hart, The University of Arizona (United States)

22 Wavefront Correctors II

Pierre-Yves Madec, European Southern Observatory (Germany)

23 Wavefront Sensing IV

Yutaka Hayano, Subaru Telescope, National Astronomical

Observatory of Japan (United States) 
Proc. of SPIE Vol. $8447844701-38$

Downloaded From: https://www.spiedigitallibrary.org/conference-proceedings-of-spie on 26 Apr 2023 Terms of Use: https://www.spiedigitallibrary.org/terms-of-use 